EUROPEAN ORGANIZATION FOR NUCLEAR RESEARCH

CERN-PPE/95-57

April 28, 1995

\title{
Measurement of the Longitudinal, Transverse and Asymmetry Fragmentation Functions at LEP
}

\author{
OPAL Collaboration
}

\begin{abstract}
The fragmentation function for the process $\mathrm{e}^{+} \mathrm{e}^{-} \longrightarrow h+X$, where $h$ represents a hadron, may be decomposed into transverse, longitudinal and asymmetric contributions by analysis of the distribution of polar production angles. A number of new tests of QCD have been proposed using these fragmentation functions, but so far no data have been published on the separate components. We have performed such a separation using data on charged particles from hadronic $\mathrm{Z}^{0}$ decays at OPAL, and have compared the results with the predictions of QCD. By integrating the fragmentation functions, we determine the average charged particle multiplicity to be $\overline{n_{c h}}=21.05 \pm 0.20$. The longitudinal to total cross-section ratio is determined to be $\sigma_{L} / \sigma_{t o t}=0.057 \pm 0.005$. From the longitudinal fragmentation function we are able to extract the gluon fragmentation function. The connection between the asymmetry fragmentation function and electroweak asymmetries is discussed.
\end{abstract}

Submitted to Zeitschrift für Physik C. 


\section{The OPAL Collaboration}

R. Akers ${ }^{16}$, G. Alexander ${ }^{23}$, J. Allison ${ }^{16}$, N. Altekamp ${ }^{5}$, K. Ametewee ${ }^{25}$, K.J. Anderson ${ }^{9}$, S. Anderson ${ }^{12}$, S. Arcelli ${ }^{2}$, S. Asai ${ }^{24}$, D. Axen ${ }^{29}$, G. Azuelos ${ }^{18, a}$, A.H. Ball ${ }^{17}$, E. Barberio ${ }^{26}$, R.J. Barlow ${ }^{16}$, R. Bartoldus ${ }^{3}$, J.R. Batley ${ }^{5}$, G. Beaudoin ${ }^{18}$, S. Bethke ${ }^{14}$, A. Beck ${ }^{23}$, G.A. Beck ${ }^{13}$, C. Beeston ${ }^{16}$, T. Behnke ${ }^{27}$, K.W. Bell ${ }^{20}$, G. Bella ${ }^{23}$, S. Bentvelsen ${ }^{8}$, P. Berlich ${ }^{10}$, J. Bechtluft ${ }^{14}$, O. Biebel ${ }^{14}$, I.J. Bloodworth ${ }^{1}$, P. Bock ${ }^{11}$, H.M. Bosch ${ }^{11}$, M. Boutemeur ${ }^{18}$, S. Braibant ${ }^{12}$, P. Bright-Thomas ${ }^{25}$, R.M. Brown ${ }^{20}$, A. Buijs ${ }^{8}$, H.J. Burckhart ${ }^{8}$, R. Bürgin ${ }^{10}$, C. Burgard ${ }^{27}$, P. Capiluppi ${ }^{2}$, R.K. Carnegie ${ }^{6}$, A.A.Carter ${ }^{13}$, J.R. Carter ${ }^{5}$, C.Y.Chang ${ }^{17}$, C. Charlesworth ${ }^{6}$,

D.G. Charlton ${ }^{1, b}$, S.L. Chu ${ }^{4}$, P.E.L. Clarke ${ }^{15}$, J.C. Clayton ${ }^{1}$, S.G. Clowes ${ }^{16}$, I. Cohen ${ }^{23}$, J.E. Conboy ${ }^{15}$, O.C. Cooke ${ }^{16}$, M. Cuffiani ${ }^{2}$, S. Dado ${ }^{22}$, C. Dallapiccola ${ }^{17}$, G.M. Dallavalle ${ }^{2}$, C. Darling ${ }^{31}$, S. De Jong ${ }^{12}$, L.A. del Pozo ${ }^{8}$, H.Deng ${ }^{17}$, M.S. Dixit ${ }^{7}$, E. do Couto e Silva ${ }^{12}$, J.E. Duboscq ${ }^{8}$, E. Duchovni ${ }^{26}$, G. Duckeck ${ }^{8}$, I.P. Duerdoth ${ }^{16}$, U.C. Dunwoody ${ }^{8}$, J.E.G.Edwards ${ }^{16}$, P.G. Estabrooks ${ }^{6}$, H.G. Evans ${ }^{9}$, F. Fabbri ${ }^{2}$, B. Fabbro ${ }^{21}$, M. Fanti ${ }^{2}$,

P. Fath ${ }^{11}$, F. Fiedler ${ }^{12}$, M. Fierro ${ }^{2}$, M. Fincke-Keeler ${ }^{28}$, H.M. Fischer ${ }^{3}$, R. Folman ${ }^{26}$, D.G. Fong ${ }^{17}$, M. Foucher ${ }^{17}$, H. Fukui ${ }^{24}$, A.Fürtjes ${ }^{8}$, P. Gagnon ${ }^{6}$, A. Gaidot ${ }^{21}$, J.W. Gary ${ }^{4}$, J. Gascon ${ }^{18}$, N.I. Geddes ${ }^{20}$, C. Geich-Gimbel ${ }^{3}$, S.W. Gensler ${ }^{9}$, F.X. Gentit ${ }^{21}$, T. Geralis ${ }^{20}$, G. Giacomelli ${ }^{2}$, P. Giacomelli ${ }^{4}$, R. Giacomelli ${ }^{2}$, V. Gibson ${ }^{5}$, W.R. Gibson ${ }^{13}$, J.D. Gillies ${ }^{20}$, J. Goldberg ${ }^{22}$, D.M. Gingrich ${ }^{30, a}$, M.J. Goodrick ${ }^{5}$, W. Gorn ${ }^{4}$, C. Grandi ${ }^{2}$, E. Gross ${ }^{26}$, G.G. Hanson ${ }^{12}$, M.Hansroul ${ }^{8}$, M. Hapke ${ }^{13}$, C.K.Hargrove ${ }^{7}$, P.A.Hart ${ }^{9}$, C.Hartmann ${ }^{3}$, M. Hauschild ${ }^{8}$, C.M. Hawkes ${ }^{8}$, R. Hawkings ${ }^{8}$, R.J.Hemingway ${ }^{6}$, G. Herten ${ }^{10}$, R.D. Heuer ${ }^{8}$, J.C. Hill ${ }^{5}$, S.J. Hillier ${ }^{8}$, T. Hilse ${ }^{10}$, P.R.Hobson ${ }^{25}$, D. Hochman ${ }^{26}$, R.J. Homer ${ }^{1}$, A.K. Honma ${ }^{28, a}$, R. Howard ${ }^{29}$, R.E. Hughes-Jones ${ }^{16}$, D.E. Hutchcroft ${ }^{5}$, P. Igo-Kemenes ${ }^{11}$, D.C. Imrie ${ }^{25}$, A. Jawahery ${ }^{17}$, P.W. Jeffreys ${ }^{20}$, H. Jeremie ${ }^{18}$, M. Jimack ${ }^{1}$, A. Joly ${ }^{18}$, M. Jones ${ }^{6}$, R.W.L. Jones ${ }^{8}$, P. Jovanovic ${ }^{1}$, D. Karlen ${ }^{6}$, J. Kanzaki ${ }^{24}$, K. Kawagoe ${ }^{24}$, T. Kawamoto ${ }^{24}$, R.K. Keeler ${ }^{28}$, R.G.Kellogg ${ }^{17}$, B.W. Kennedy ${ }^{20}$, B.J. King ${ }^{8}$, J. King ${ }^{13}$, J. Kirk ${ }^{29}$, S. Kluth ${ }^{5}$, T. Kobayashi ${ }^{24}$,

M. Kobel ${ }^{10}$, D.S. Koetke ${ }^{6}$, T.P. Kokott ${ }^{3}$, S. Komamiya $^{24}$, R. Kowalewski ${ }^{8}$, T. Kress $^{11}$,

P. Krieger ${ }^{6}$, J. von Krogh ${ }^{11}$, P. Kyberd ${ }^{13}$, G.D. Lafferty ${ }^{16}$, H. Lafoux ${ }^{8}$, R. Lahmann ${ }^{17}$, W.P. Lai ${ }^{19}$, D. Lanske ${ }^{14}$, J. Lauber ${ }^{8}$, J.G. Layter ${ }^{4}$, A.M. Lee ${ }^{31}$, E. Lefebvre ${ }^{18}$, D. Lellouch ${ }^{26}$,

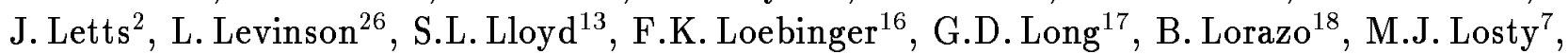
J.Ludwig ${ }^{10}$, A. Luig ${ }^{10}$, A. Malik ${ }^{21}$, M. Mannelli ${ }^{8}$, S. Marcellini ${ }^{2}$, C. Markus ${ }^{3}$, A.J. Martin ${ }^{13}$, J.P. Martin ${ }^{18}$, T. Mashimo ${ }^{24}$, W. Matthews ${ }^{25}$, P. Mättig ${ }^{3}$, J. McKenna ${ }^{29}$, E.A. Mckigney ${ }^{15}$, T.J. McMahon ${ }^{1}$, A.I. McNab ${ }^{13}$, F. Meijers ${ }^{8}$, S. Menke ${ }^{3}$, F.S. Merritt ${ }^{9}$, H. Mes ${ }^{7}$, A. Michelini ${ }^{8}$, G. Mikenberg ${ }^{26}$, D.J. Miller ${ }^{15}$, R. Mir $^{26}$, W. Mohr ${ }^{10}$, A. Montanari ${ }^{2}$, T. Mori ${ }^{24}$, M. Morii ${ }^{24}$, U. Müller ${ }^{3}$, B. Nellen ${ }^{3}$, B. Nijjhar ${ }^{16}$, S.W. O'Neale' ${ }^{1}$, F.G. Oakham ${ }^{7}$, F. Odorici ${ }^{2}$, H.O. Ogren ${ }^{12}$, N.J. Oldershaw ${ }^{16}$, C.J. Oram ${ }^{28, a}$, M.J. Oreglia ${ }^{9}$, S. Orito ${ }^{24}$, F. Palmonari ${ }^{2}$, J.P. Pansart ${ }^{21}$,

G.N.Patrick ${ }^{20}$, M.J. Pearce ${ }^{1}$, P.D. Phillips ${ }^{16}$, J.E. Pilcher ${ }^{9}$, J. Pinfold ${ }^{30}$, D.E. Plane ${ }^{8}$, P. Poffenberger ${ }^{28}$, B. Poli ${ }^{2}$, A.Posthaus ${ }^{3}$, T.W. Pritchard ${ }^{13}$, H. Przysiezniak ${ }^{30}$,

M.W. Redmond ${ }^{8}$, D.L. Rees ${ }^{1}$, D. Rigby ${ }^{1}$, M.G. Rison ${ }^{5}$, S.A. Robins ${ }^{13}$, N. Rodning ${ }^{30}$, J.M. Roney ${ }^{28}$, E. $\operatorname{Ros}^{8}$, A.M. Rossi ${ }^{2}$, M. Rosvick ${ }^{28}$, P. Routenburg ${ }^{30}$, Y. Rozen ${ }^{8}$, K. Runge ${ }^{10}$, O. Runolfsson ${ }^{8}$, D.R. Rust ${ }^{12}$, M. Sasaki ${ }^{24}$, C. Sbarra ${ }^{2}$, A.D. Schaile ${ }^{8}$, O. Schaile ${ }^{10}$, F.Scharf ${ }^{3}$, P.Scharff-Hansen ${ }^{8}$, P.Schenk ${ }^{4}$, B. Schmitt ${ }^{3}$, M.Schröder ${ }^{8}$, H.C.Schultz-Coulon ${ }^{10}$, P. Schütz ${ }^{3}$, M. Schulz ${ }^{8}$, J.Schwiening ${ }^{3}$, W.G. Scott ${ }^{20}$, M.Settles ${ }^{12}$, T.G.Shears ${ }^{16}$, B.C.Shen ${ }^{4}$, C.H.Shepherd-Themistocleous ${ }^{7}$, P.Sherwood ${ }^{15}$, G.P.Siroli ${ }^{2}$, A.Skillman ${ }^{15}$, A.Skuja ${ }^{17}$, A.M.Smith ${ }^{8}$, T.J.Smith ${ }^{28}$, G.A.Snow ${ }^{17}$, R. Sobie ${ }^{28}$, S.Söldner-Rembold ${ }^{10}$, R.W. Springer ${ }^{30}$, M.Sproston ${ }^{20}$, A.Stahl ${ }^{3}$, M.Starks ${ }^{12}$, C.Stegmann ${ }^{10}$, K.Stephens ${ }^{16}$, J.Steuerer ${ }^{28}$,

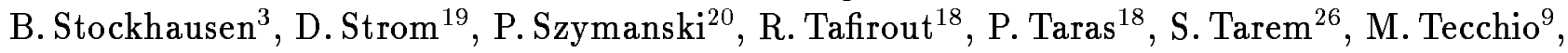




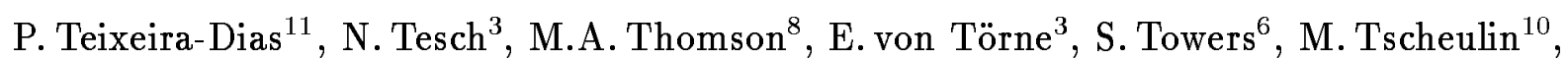
T. Tsukamoto ${ }^{24}$, A.S. Turcot ${ }^{9}$, M.F. Turner-Watson ${ }^{8}$, P. Utzat ${ }^{11}$, R. Van Kooten ${ }^{12}$, G. Vasseur ${ }^{21}$, P. Vikas ${ }^{18}$, M. Vincter ${ }^{28}$, F. Wäckerle ${ }^{10}$, A. Wagner ${ }^{27}$, D.L. Wagner ${ }^{9}$, C.P. Ward ${ }^{5}$, D.R. Ward ${ }^{5}$, J.J. Ward ${ }^{15}$, P.M. Watkins ${ }^{1}$, A.T. Watson ${ }^{1}$, N.K. Watson ${ }^{7}$, P. Weber ${ }^{6}$, P.S. Wells ${ }^{8}$, N. Wermes ${ }^{3}$, B. Wilkens ${ }^{10}$, G.W.Wilson ${ }^{27}$, J.A.Wilson ${ }^{1}$, T. Wlodek ${ }^{26}$, G. Wolf ${ }^{26}$, S. Wotton ${ }^{11}$, T.R. Wyatt ${ }^{16}$, G. Yekutielii ${ }^{26}$, V. Zacek ${ }^{18}$, W.Zeuner ${ }^{8}$, G.T. Zorn ${ }^{17}$.

${ }^{1}$ School of Physics and Space Research, University of Birmingham, Birmingham B15 2TT, UK 'Dipartimento di Fisica dell' Università di Bologna and INFN, I-40126 Bologna, Italy

${ }^{3}$ Physikalisches Institut, Universität Bonn, D-53115 Bonn, Germany

${ }^{4}$ Department of Physics, University of California, Riverside CA 92521, USA

${ }^{5}$ Cavendish Laboratory, Cambridge CB3 0HE, UK

${ }^{6}$ Carleton University, Department of Physics, Colonel By Drive, Ottawa, Ontario K1S 5B6, Canada

${ }^{7}$ Centre for Research in Particle Physics, Carleton University, Ottawa, Ontario K1S 5B6, Canada

${ }^{8}$ CERN, European Organisation for Particle Physics, CH-1211 Geneva 23, Switzerland

${ }^{9}$ Enrico Fermi Institute and Department of Physics, University of Chicago, Chicago IL 60637, USA

${ }^{10}$ Fakultät für Physik, Albert Ludwigs Universität, D-79104 Freiburg, Germany

${ }^{11}$ Physikalisches Institut, Universität Heidelberg, D-69120 Heidelberg, Germany

${ }^{12}$ Indiana University, Department of Physics, Swain Hall West 117, Bloomington IN 47405, USA

${ }^{13}$ Queen Mary and Westfield College, University of London, London E1 4NS, UK

${ }^{14}$ Technische Hochschule Aachen, III Physikalisches Institut, Sommerfeldstrasse 26-28, D-52056 Aachen, Germany

${ }^{15}$ University College London, London WC1E 6BT, UK

${ }^{16}$ Department of Physics, Schuster Laboratory, The University, Manchester M13 9PL, UK

${ }^{17}$ Department of Physics, University of Maryland, College Park, MD 20742, USA

${ }^{18}$ Laboratoire de Physique Nucléaire, Université de Montréal, Montréal, Quebec H3C 3J7, Canada

${ }^{19}$ University of Oregon, Department of Physics, Eugene OR 97403, USA

${ }^{20}$ Rutherford Appleton Laboratory, Chilton, Didcot, Oxfordshire OX11 0QX, UK

${ }^{21}$ CEA, DAPNIA/SPP, CE-Saclay, F-91191 Gif-sur-Yvette, France

${ }^{22}$ Department of Physics, Technion-Israel Institute of Technology, Haifa 32000, Israel

${ }^{23}$ Department of Physics and Astronomy, Tel Aviv University, Tel Aviv 69978, Israel

${ }^{24}$ International Centre for Elementary Particle Physics and Department of Physics, University of Tokyo, Tokyo 113, and Kobe University, Kobe 657, Japan

${ }^{25}$ Brunel University, Uxbridge, Middlesex UB8 3PH, UK

${ }^{26}$ Particle Physics Department, Weizmann Institute of Science, Rehovot 76100, Israel

${ }^{27}$ Universität Hamburg/DESY, II Institut für Experimental Physik, Notkestrasse 85, D-22607

Hamburg, Germany

${ }^{28}$ University of Victoria, Department of Physics, P O Box 3055, Victoria BC V8W 3P6, Canada

${ }^{29}$ University of British Columbia, Department of Physics, Vancouver BC V6T 1Z1, Canada

${ }^{30}$ University of Alberta, Department of Physics, Edmonton AB T6G 2J1, Canada

${ }^{31}$ Duke University, Dept of Physics, Durham, NC 27708-0305, USA 
${ }^{a}$ Also at TRIUMF, Vancouver, Canada V6T 2A3

${ }^{b}$ Royal Society University Research Fellow 


\section{Introduction}

The fragmentation function for the process $\mathrm{e}^{+} \mathrm{e}^{-} \longrightarrow h+X$, where $h$ represents a hadron, is defined as $\left(1 / \sigma_{t o t}\right) d \sigma^{h} / d x$, where $x$ may be either the fractional momentum, $x_{p}=2 p / E_{c m}$, or the fractional energy, $x_{E}=2 E / E_{c m}$, carried by the hadron, $E_{c m}$ is the centre of mass energy and $\sigma_{t o t}$ is the total hadronic cross-section. Although perturbative QCD is unable to predict the form of such fragmentation functions, it can predict how they should evolve with $E_{c m}[1,2]$. Such scaling violations may, for example, form the basis of a measurement of the strong coupling constant $\alpha_{s}[3]$.

It can be shown $[4,2]$ that the most general form of the differential cross-section for the production of a hadron $h$ via a spin one $\mathrm{Z}^{0}$ or photon is (for the case of unpolarized $\mathrm{e}^{ \pm}$beams, and averaging over polarizations of $h$ ):

$$
\frac{d^{2} \sigma^{h}}{d x d \cos \theta}=\frac{3}{8}\left(1+\cos ^{2} \theta\right) \frac{d \sigma_{T}^{h}}{d x}+\frac{3}{4} \sin ^{2} \theta \frac{d \sigma_{L}^{h}}{d x}+\frac{3}{4} \cos \theta \frac{d \sigma_{A}^{h}}{d x}
$$

where $\theta$ is the angle between the hadron and the $\mathrm{e}^{-}$beam. These three contributions to the fragmentation function are referred to as the transverse, longitudinal and asymmetric parts. By analysis of the distribution of production angles $\theta$ for any value of $x$, the fragmentation function may therefore be decomposed into its longitudinal, transverse and asymmetric components.

A number of new tests of QCD have been proposed using these fragmentation functions [2], which makes them of interest beyond their relevance to studies of scaling violations. For example, in the absence of QCD radiation, and assuming standard model couplings for the $Z^{0} / \gamma$, the $\mathrm{Z}^{0} / \gamma$ are produced only in transverse polarization states and the longitudinal fragmentation function should be zero. The leading contribution to the longitudinal fragmentation function is therefore proportional to $\alpha_{s}$, and a measurement of the longitudinal fragmentation function at small values of $x$ is expected to be particularly sensitive to the gluon fragmentation function. This study is therefore complementary to other OPAL studies of gluon fragmentation, which have been based on tagged three-jet events $[5,6]$. The asymmetry term represents parity violating effects, which are expected to arise in the electroweak interaction.

So far no experimental data have been published on the separate transverse, longitudinal and asymmetry components. In the present study we perform such a separation of the fragmentation function using data on hadronic $\mathrm{Z}^{0}$ decays from OPAL. In essence this involves measuring the hadron production as a function of $\cos \theta$ at many values of $x$. The procedure, and the assessment of systematic uncertainties, are discussed in Sections 2 and 3. In Section 4 we compare the results, and in particular the longitudinal and transverse cross-sections which may be obtained by integrating the fragmentation functions, with the predictions of QCD. We also investigate the possibility of inferring the gluon fragmentation function from the data, and discuss the relationship between the asymmetry fragmentation function and electroweak asymmetries in $\mathrm{Z}^{0}$ decays. 


\section{Experimental Procedure}

The OPAL detector has been described in detail elsewhere [7]. The present analysis depends on measuring the momenta and production angles of charged particles, and therefore relies almost entirely on the central tracking system. This consists of a silicon microvertex detector [8] and three drift chamber systems, all of which lie within an axial magnetic field of $0.435 \mathrm{~T}$. A precision vertex drift chamber, of outer radius $24 \mathrm{~cm}$ and a length of $100 \mathrm{~cm}$, provides space points with a resolution of about $50 \mu \mathrm{m}$ in the $r-\phi$ plane $\mathrm{e}^{*}$. The vertex detector contains 12 axial wires per $\phi$-sector, followed by 6 stereo wires, inclined at an angle of $\sim 4^{\circ}$ to the $z$-axis to improve the $z$-resolution. Surrounding this is a large jet chamber, of outer radius $185 \mathrm{~cm}$ and length about $400 \mathrm{~cm}$, which provides up to 159 digitizations per track with an $r$ - $\phi$ resolution of around $130 \mu \mathrm{m}$. Using charge division a resolution of about $6 \mathrm{~cm}$ in $z$ may be obtained for each wire in multihadronic events. On the outside lies a system of z-chambers - thin drift chambers, with a resolution of about $300 \mu \mathrm{m}$ in $z$, which serve to improve the determination of $\theta$, as discussed below. In addition, the OPAL detector contains an electromagnetic calorimeter based on lead glass and equipped with presamplers, a hadronic calorimeter formed by instrumenting the iron return yoke of the magnet, all surrounded by a muon detection system.

Hadronic events were selected for this analysis using standard cuts as described in Refs. [9, 10]. The number of charged tracks was required to be at least seven, in order to reduce $\tau^{+} \tau^{-}$and two-photon background. The thrust axis [11] was determined using clusters of energy in the lead glass electromagnetic calorimeter, and was required to satisfy $|\cos \theta|<0.95$. This cut was based purely on the calorimetry in order to reduce any bias to the track information used for the main analysis. Under these criteria, 3056609 events were selected from the data recorded by OPAL from 1990 to 1994 . Of these, approximately $90 \%$ were recorded at the $\mathrm{Z}^{0}$ peak, and the remainder at c.m. energies within $\pm 3 \mathrm{GeV}$; the inclusion of off-peak data has a negligible influence on the results. Samples of Monte Carlo events (based on the JETSET 7.3 parton shower model [12]) processed through the OPAL detector simulation program [13] were used to correct for detector resolution, acceptance and initial state radiation; in total 3733725 Monte Carlo events were used, of which $94.1 \%$ satisfied the event selection criteria.

Tracks to be used in the analysis were selected according to the following criteria: momentum greater than $0.1 \mathrm{GeV} / c$, at least 20 reconstructed points in the jet chamber, extrapolation to the nominal collision point within $2 \mathrm{~cm}$ in $r-\phi$ and $25 \mathrm{~cm}$ in $z$ and measured momentum less than $65 \mathrm{GeV} / c$. With these criteria, 20.0 tracks were selected per event on average. Cuts were then imposed on the azimuthal position of tracks (extrapolated to the point of exit from the jet chamber) in order to eliminate tracks pointing at gaps between $z$-chamber panels or at $\mathrm{z}$-chambers which were inactive for significant periods of time; these cuts removed $24.4 \%$ of the accepted tracks, but significantly increased the likelihood of a track having z-chamber information.

The present analysis depends upon measurements of the momenta and polar angles of charged tracks. The resolution on the momentum in the $r-\phi$ plane is given by $\sigma_{p_{T}} / p_{T}=$ $\sqrt{0.020^{2}+\left(0.0015 p_{T} /(\mathrm{GeV} / c)\right)^{2}}$ for tracks in the barrel region of the detector. In order to improve the resolution in $\cos \theta$, tracks were constrained to emanate from the primary vertex

${ }^{*}$ The OPAL coordinate system is defined so that $z$ is the coordinate parallel to the $\mathrm{e}^{-}$beam, $r$ is the coordinate normal to this axis, $\theta$ is the polar angle with respect to $z$ and $\phi$ is the azimuthal angle about the $z$-axis. 
point in $z$, within its errors. With this constraint the resolution in $\cos \theta$ for tracks which also had hits in the $\mathrm{z}$-chambers was approximately 0.001 , averaged over all momenta. After the cut on $\phi$ mentioned above, approximately $89 \%$ of tracks in the region $|\cos \theta|<0.64$ have hits in the z-chamber system, a figure which rises to $93 \%$ when tracks having $x_{E}>0.01$ are considered. For tracks passing through the ends of the jet chamber (roughly $|\cos \theta|>0.74$ ), the end of the last wire which registered a hit in the jet chamber may be taken to be the point at which the track exited from the chamber. The measurement of $\cos \theta$ may be significantly improved by using this endpoint information. Approximately $80 \%$ of tracks in this region have such information, rising to $90 \%$ for $x_{E}>0.01$, with a resolution in $\cos \theta$ of about 0.001 . For the $\sim 16 \%$ of tracks which have neither z-chamber nor endpoint information, the determination of $\theta$ comes mainly from charge division measurements in the jet chamber, yielding a resolution in $\cos \theta$ of roughly 0.01; also these charge division measurements are relatively susceptible to systematic problems. For about one third of these tracks in the region $|\cos \theta|<0.75$, further information is available from the barrel presampler detector; this only provides a small improvement in the resolution, but is expected to reduce systematic uncertainties.

Distributions of $\cos \theta$ were formed for each of 22 bins of $x_{E}$, the pion mass being assumed in computing $E$. The bins are specified in Table 1 . In order to be sensitive to the asymmetry term, we multiply $\cos \theta$ by the particle charge, $q$. The width of the bins in $x_{E}$ is much greater than the expected resolution. The bin width in $\cos \theta$ was chosen to be 0.01 , which is well matched to the poorer resolution of the $16 \%$ of tracks which have neither z-chamber nor endpoint information. The data in each range of $x_{E}$ were then corrected for experimental acceptance and resolution using Monte Carlo events, as follows. We define

$$
g(x, q \cdot \cos \theta)=\frac{1}{N} \frac{\Delta n}{\Delta x \Delta(q \cdot \cos \theta)}
$$

where $N$ is the total number of events and $\Delta n$ the number of tracks in a bin of widths $\Delta x$ and $\Delta(q \cdot \cos \theta)$. We then calculate $g_{\text {det }}(x, q \cdot \cos \theta)$ from the Monte Carlo sample including detector simulation using events and tracks satisfying the same cuts as used for the data ("detector level"), and $g_{\text {had }}(x, q \cdot \cos \theta)$ for all stable charged particles in all events without detector simulation and without initial state radiation ("hadron level"). In this context, particles having mean lifetimes shorter than $3 \times 10^{-10} \mathrm{~s}$ are deemed to be unstable. At the hadron level, the true mass from the Monte Carlo is used in computing $x_{E}$. The value of $g_{o b s}(x, q \cdot \cos \theta)$ from the observed data is then corrected by multiplying by the ratio $g_{\text {had }}(x, q \cdot \cos \theta) / g_{\text {det }}(x, q \cdot \cos \theta)$ inferred from the Monte Carlo. In order to estimate the statistical errors on the correction factors, taking account of correlations between the numerator and denominator, we have divided the Monte Carlo sample into independent subsets, and examined the differences between the corrections inferred from each subset.

\section{Extraction of Fragmentation Functions}

The analysis is based on all charged particles satisfying the above cuts. Fig. 1 shows a corrected $q \cdot \cos \theta$ distribution for a typical bin of $x_{E}$, together with the correction factors. The azimuthal cut removes $24.2 \%$ of the geometrical acceptance, and the correction anticipated on the basis of this cut alone is shown by the horizontal line in fig. 1(b). In the barrel region, roughly 
$|\cos \theta|<0.6$, the correction factors are rather uniform, and close to the expected level. Around $|\cos \theta|=0.7$ they exhibit some structure, and the corrected distribution is not smooth. This corresponds to a gap in acceptance between the z-chamber system and the endpoint region in this gap the tracks have significantly poorer resolution. Although these effects are largely simulated by the Monte Carlo, the structure in the corrected data shows that there remain systematic problems in this region which are imperfectly simulated. In the endcap region, $|\cos \theta|>0.8$, the correction factors rise with $|\cos \theta|$ as a consequence of the experimental cuts. Nevertheless, it is desirable to extend the analysis into the endcaps if possible, in order to reduce the statistical errors. The analysis depends on distinguishing the $\left(1+\cos ^{2} \theta\right)$ and $\sin ^{2} \theta$ components of the angular distribution, and these components differ most in the endcaps.

The transverse, longitudinal and asymmetric components of the fragmentation functions are then inferred by fitting the corrected $g(x, q \cdot \cos \theta)$ distribution in each bin of $x_{E}$ to the function:

$$
g(x, q \cdot \cos \theta)=\frac{3}{8}\left(1+\cos ^{2} \theta\right) F_{T}(x)+\frac{3}{4} \sin ^{2} \theta F_{L}(x)+\frac{3}{4} q \cdot \cos \theta F_{A}(x),
$$

where $F_{T}(x)=\left(1 / \sigma_{t o t}\right) d \sigma_{T}^{c h} / d x$ etc, the superscript ch implying the sum over all charged particles. By default we fit in the region $|\cos \theta|<0.9$, since the correction factors at greater values of $|\cos \theta|$ are large, and we eliminate the region $0.64<|\cos \theta|<0.76$ where systematic effects are seen to be significant; 156 bins in $\cos \theta$ therefore remain to be used in the fits. Alternative fit regions are considered as systematic checks on the results. We show in Fig. 2 the result of a fit to two typical ranges of $x_{E}, 0.04<x_{E}<0.05$ and $0.20<x_{E}<0.25$, together with the residuals of the fit. Bins eliminated from the fit are shown with zero errors. The fits are reasonably satisfactory, having $\chi^{2} /$ d.o.f. $=213 / 153$ and $210 / 153$ respectively, and the residual plots show no undesirable structure. We note that the bin at lower $x_{E}$ has a sizeable longitudinal component, while the asymmetry term is very close to zero. At higher $x_{E}$ the longitudinal term becomes proportionally smaller, while the asymmetry becomes perceptible.

Considering the fits in the 22 regions of $x_{E}$, the poorest fit by far occurs for the bin at smallest $x_{E}, x_{E}<0.01$, where $\chi^{2} /$ d.o.f. $=8.0$. In this bin the measurement of $\cos \theta$ is significantly worse because of a small proportion of $z$-chamber and endpoint measurements. This bin contributes relatively little to the subsequent analysis, and is assigned a rather large systematic error. Amongst the other bins of $x_{E}$ the average value of $\chi^{2} /$ d.o.f. is 1.36 . Since the values of $\chi^{2} /$ d.o.f. for all bins of $x_{E}$ tend to be slightly greater than unity, this probably indicates the presence of small systematic effects in the data which are not corrected by the Monte Carlo, as discussed below.

The values of $F_{T}(x), F_{L}(x)$ and $F_{A}(x)$ derived from the data are listed in Table 1, together with their statistical errors. We also give values for the transverse, longitudinal and asymmetry cross-sections, which are defined through an energy conservation sum rule:

$$
\sigma_{T}=\sum_{h} \int_{0}^{1} d x \frac{x}{2} \frac{d \sigma_{T}^{h}}{d x}
$$

and similarly for $\sigma_{L}$ and $\sigma_{A}$. Clearly, $\sigma_{T}+\sigma_{L}=\sigma_{t o t}$, since the sum of the values of $x / 2$ over all particles in an event must equal unity. In the present analysis we only measure charged hadrons, so we refer to the cross-sections as $\sigma_{T}^{c h}, \sigma_{L}^{c h}$ and $\sigma_{A}^{c h}$, and they are measured as a fraction of the total hadronic cross-section $\sigma_{t o t}$. In order to perform the integration, we take 
the average value of $x$ for each bin from the JETSET or HERWIG[14] Monte Carlo programs (which yield consistent values). We note that the values obtained for $F_{L}(x)$ become negative for $x>0.5$, however they are consistent with zero within errors.

It is next necessary to assess the likely systematic uncertainties on these measurements, which, in view of the systematic effects noted above, may be expected to be significant. We use two basic approaches - we vary the region of $q \cdot \cos \theta$ over which the data are fitted so as to exclude various regions where problems may be expected, or we change the event and track selection criteria.

\section{Variation of fit region}

We consider the following alternative fit regions for the standard data sample:

- We remove the data points close to the edges of the standard fit region, by demanding $|\cos \theta|<0.88$ and also excluding a wider region $0.60<|\cos \theta|<0.80$.

- We restrict the analysis to the barrel region, $|\cos \theta|<0.64$. The statistical errors are roughly doubled by this restriction.

- We restrict the analysis more tightly to the barrel region, $|\cos \theta|<0.60$.

We take as a systematic error the largest change seen under any of the above conditions.

\section{Variation of selections}

We vary the following cuts:

- The cut on the distance of closest approach to the collision point in $r$ - $\phi$ was tightened to $\left|d_{0}\right|<1 \mathrm{~cm}$ or loosened to $\left|d_{0}\right|<5 \mathrm{~cm}$.

- The cut on the $z$-coordinate at the point of closest approach to the collision point in $r$ - $\phi$ was tightened to $\left|z_{0}\right|<10 \mathrm{~cm}$.

- The minimum track momentum cut was replaced by a requirement that the momentum component in the $r-\phi$ plane be greater than $0.15 \mathrm{GeV} / \mathrm{c}$.

- The cut on the number of jet chamber hits, $N_{C J}$, was tightened to 40 .

- The cut on the thrust axis was changed to $|\cos \theta|<0.90$.

- The barrel presampler information was not used.

- For the data recorded in 1993-4 the microvertex detector was upgraded to provide $z$-coordinate readout. These $z$-measurements were not used by default, but the analysis was repeated with them included where available.

In order to be conservative, the changes resulting from each of these modifications to the analysis were combined in quadrature to obtain an estimate of the systematic uncertainty.

The total error quoted in Table 1 was obtained by combining the statistical, fit range and selection cuts errors in quadrature. The systematic error may be seen to be significantly larger than the statistical uncertainty for the longitudinal and transverse fragmentation functions, while for the asymmetry term the two are comparable. The accuracy of measurements of $F_{L}(x)$ is typically $10 \%$ in the region $0.02<x_{E}<0.1$, and the error becomes comparable with the measurement for $x_{E}>\mathbf{0 . 3}$. The longitudinal cross-section, $\sigma_{L}^{c h}$ is determined to an accuracy of 
about $9 \%$. In Table 2 we show a detailed breakdown of the systematic error contributions to $\sigma_{T}^{c h}, \sigma_{L}^{c h}$ and $\sigma_{A}^{c h}$.

As a check on the control of systematic effects it is instructive to derive $F_{T}(x), F_{L}(x)$ and $F_{A}(x)$ from each year of data separately. We find that there are significant differences if only statistical errors are considered. However, the data are compatible within the systematic errors we have estimated, and the final values quoted in Table 1 are consistent with all separate years' results within the systematic errors.

We have also made a check in which $\cos \theta$ is not multiplied by the particle charge, and the analysis repeated. In this case the asymmetry for positive and negative particles should cancel. We find that the fits yield values for $F_{A}(x)$ which are consistent with zero within the statistical errors, while the values of $F_{T}(x)$ and $F_{L}(x)$ do not differ significantly from the standard results. The regions around the z-chamber cathodes (every $50 \mathrm{~cm}$ in $z$ ) are found to contribute significantly to the large values of $\chi^{2} /$ d.o.f. noted above; eliminating these regions reduces $\chi^{2} /$ d.o.f., with a negligible influence on the fitted parameters.

We note that the average charged particle multiplicity should be given by the integral of the transverse and longitudinal fragmentation functions, i.e. $\overline{n_{c h}}=\int_{0}^{1}\left[\frac{d \sigma_{T}^{c h}}{d x}+\frac{d \sigma_{L}^{c h}}{d x}\right] d x$. From the data in Table 1 we obtain $\overline{n_{c h}}=21.05 \pm 0.01 \pm 0.20$. This measurement is in agreement with a previous OPAL determination of $21.40 \pm 0.02 \pm 0.43[15]$ based on the measurement of the charged multiplicity distribution, and has a smaller uncertainty. The systematic error includes an additional small contribution for this measurement arising from the use of the HERWIG model instead of JETSET for the corrections, in order to be compatible with Ref. [15].

\section{Comparisons with QCD}

\subsection{Comparison with QCD Monte Carlo models}

In Fig. 3 we show the measurements of $F_{T}(x)$ and $F_{L}(x)$ (for charged particles) as a function of $x_{E}$, including their systematic errors as given in Table 1 . We note that the longitudinal fragmentation function $F_{L}(x)$ falls much more rapidly with $x_{E}$ than the transverse fragmentation function. For comparison, we show the predictions of the parton shower models, JETSET and HERWIG ${ }^{\dagger}$. The fragmentation functions for the models were separated using the weighting method described in Ref. [2] $]^{\ddagger}$. The measurements of $F_{T}(x)$ are in reasonable agreement with both models over most of the range of $x_{E}$, though some discrepancies are seen for $x_{E}>0.6$. In the case of $F_{L}(x)$ the data are compatible with both models within the errors, which are strongly correlated.

\footnotetext{
${ }^{\dagger}$ The parameters of the models were deduced from fits to global event shapes, as described in Ref. [16]. We used JETSET version 7.3 with parameters as given in Ref. [16] and HERwIG version 5.6 with parameters given in Ref. [5].

\$The transverse component of the fragmentation function may be projected out by weighting each particle by $\left(5 \cos ^{2} \theta-1\right)$ while the appropriate weight function for the longitudinal component is $\left(2-5 \cos ^{2} \theta\right)$ and for the asymmetry component is $(2 \cos \theta)$.
} 
In Fig. 4 we show the measurements of $F_{A}(x)$ (for positively charged particles plus negative particles reflected about $\cos \theta=0$ ) as a function of $x_{E}$, including their systematic errors as given in Table 1. The measurements only differ significantly from zero for $x_{E}>0.15$. For comparison, we again show the predictions of the JETSET and HERWIG models. The model calculations, based on 10 million events, have significant statistical errors, indicated by the fluctuations in the curves, but they agree well with the data, especially in the case of JETSET. The measured value of the integrated asymmetry cross-section, $\sigma_{A}^{c h}=-0.0037 \pm 0.0007$, agrees with the prediction of JETSET $(-0.0041 \pm \mathbf{0 . 0 0 0 1})$, though it is somewhat smaller in magnitude than the expectation from Herwig $(-0.0055 \pm 0.0001)$. We discuss $F_{A}(x)$ further in Sect. 4.4 .

\subsection{The Longitudinal Cross-section}

There are QCD predictions [2] for the longitudinal and transverse cross-sections to $\mathcal{O}\left(\alpha_{s}\right)$ :

$$
\sigma_{T}=\sigma_{0} \quad ; \quad \sigma_{L}=\left(\frac{\alpha_{s}}{\pi}\right) \sigma_{0}
$$

where $\sigma_{0}$ is the Born cross-section, i.e. the hadronic cross-section in the absence of QCD radiation. Thus the whole of the leading order QCD correction to the total cross-section appears in $\sigma_{L}$. The higher order terms are not yet known. It is known [17] that the $\mathcal{O}\left(\alpha_{s}^{2}\right)$ and $\mathcal{O}\left(\alpha_{s}^{3}\right)$ contributions to the total hadronic cross-section are relatively small, $\sim 3 \%$ and $\sim-2 \%$ of the $\mathcal{O}\left(\alpha_{s}\right)$ contribution respectively. Our measurements of $\sigma_{L}$ and $\sigma_{T}$ are based on charged particles only; to compare with the calculations we need to correct for the effect of neutrals. To do this, we assume that the ratio of the charged to neutral contributions are the same for $\sigma_{T}$ and $\sigma_{L}$. This assumption is verified, to a precision much better than the experimental uncertainties, by the JETSET model, which yields $\sigma_{T}^{c h} / \sigma_{T}=0.6150 \pm 0.0001$ and $\sigma_{L}^{c h} / \sigma_{L}=0.609 \pm 0.002$ and by HERWIG which gives $\sigma_{T}^{c h} / \sigma_{T}=0.6197 \pm 0.0001$ and $\sigma_{L}^{c h} / \sigma_{L}=0.616 \pm 0.002$. We therefore multiply our measured values of $\sigma_{T}^{c h}$ and $\sigma_{L}^{c h}$ by the factor $\frac{\sigma_{T}+\sigma_{L}}{\sigma_{T}^{c h}+\sigma_{L}^{c h}}=\frac{\sigma_{t o t}}{\sigma_{T}^{c h}+\sigma_{L}^{c h}}=1 / 0.623$ determined from data. After taking account of systematic uncertainties as before we obtain:

$$
\begin{aligned}
& \sigma_{T} / \sigma_{t o t}=0.943 \pm 0.005 \\
& \sigma_{L} / \sigma_{t o t}=0.057 \pm 0.005
\end{aligned}
$$

These may be converted to measurements of $\sigma_{T} / \sigma_{0}$ and $\sigma_{L} / \sigma_{0}$, using measurements of the $\mathrm{Z}^{0}$ lineshape [18] which yield $\sigma_{t o t} / \sigma_{0}=1.043 \pm 0.002$.

Taking the value of $\alpha_{s}=0.123 \pm 0.006$ [19] which is derived from jet rates and global event shapes, and which also describes well the total hadronic cross-section [17], we would expect $\sigma_{T} / \sigma_{t o t}=0.962 \pm 0.002$ and $\sigma_{L} / \sigma_{t o t}=0.038 \pm 0.002$. The data therefore indicate that higher order corrections to the longitudinal and transverse cross-sections and/or hadronization effects are not negligible. In Fig. 5 we show the predictions of the QCD Monte Carlo program JETSET for the longitudinal cross-section $\sigma_{L} / \sigma_{t o t}$ as a function of centre-of-mass energy. The predictions of the JETSET parton shower model are shown for the produced hadrons and for the partons at the end of the parton shower. The difference between these gives an indication of the influence of hadronization effects, which clearly fall rapidly with increasing centre-of-mass energy, but which are not completely negligible at the $\mathrm{Z}^{0}$ mass. The contribution of non-perturbative (hadronization) effects to the ratio $\sigma_{L} / \sigma_{t o t}$ is expected to exhibit a power behaviour of the form 
$1 / E_{c m}$ - estimates have been made in Ref. [20], yielding approximately $(0.8 \mathrm{GeV}) / E_{c m}$, and in Ref. [21], giving $(0.9 \pm 0.1 \mathrm{GeV}) / E_{c m}$, in good agreement with the expectation from JETSET. The HERWIG model gives similar results. The predicted values of $\sigma_{L} / \sigma_{t o t}$ at $E_{c m}=M_{\mathrm{Z}^{\circ}}$ for the two models are:

$$
\begin{array}{ll}
\text { JETSET parton shower }- \text { hadron level } & 0.0592 \pm \mathbf{0 . 0 0 0 4} \\
\text { JETSET parton shower }- \text { parton level } & 0.0519 \pm 0.0004 \\
\text { HERWIG parton shower - hadron level } & 0.0564 \pm \mathbf{0 . 0 0 0 4} \\
\text { HERWIG parton shower - parton level } & 0.0485 \pm \mathbf{0 . 0 0 0 4}
\end{array}
$$

The experimental value of $\sigma_{L} / \sigma_{\text {tot }}=0.057 \pm 0.005$ therefore lies very close to the model expectations at the hadron level. It appears that both hadronization and $\mathcal{O}\left(\alpha_{s}^{2}\right)$ and higher order perturbative effects contribute significantly to the difference between the $\mathcal{O}\left(\alpha_{s}\right)$ prediction and the observed value. The influence of heavy quark mass effects on $\sigma_{L}$ has been estimated in Ref. [2] to be of order $1.2\left(m_{q} / E_{c m}\right)^{2}$, where $m_{q}$ is the quark mass, and may thus be expected to be small at LEP energies.

\subsection{The Gluon Fragmentation Function}

As discussed in Ref. [2], the longitudinal fragmentation function may be related to the gluon fragmentation function $D_{g}(z)$, where $z$ is the fraction of the energy of a gluon carried by a hadron:

$$
F_{L}(x)=\frac{\alpha_{s}}{2 \pi} C_{F} \int_{x}^{1} \frac{d z}{z}\left[F_{T}(z)+4\left(\frac{z}{x}-1\right) D_{g}(z)\right]+\mathcal{O}\left(\alpha_{s}^{2}\right),
$$

where the colour factor $C_{F}=\frac{4}{3}$. At large values of $x$ (where our measurements are poor) $F_{L}(x)$ is expected to be dominated by the $F_{T}$ term in the integral (representing the quark contribution), which we have measured. The contribution from the gluon fragmentation $D_{g}$ is greatest at small values of $x$, where our data are most significant, and we may therefore hope to make a useful measurement of $D_{g}(z)$ at small values of $z$. We choose to extract $D_{g}(z)$ (for charged particles) in the following way. We define a new function $F_{g}(x)$

$$
F_{g}(x) \equiv \frac{\pi}{2 \alpha_{s} C_{F}} F_{L}(x)-\frac{1}{4} \int_{x}^{1} \frac{F_{T}(z)}{z} d z=\int_{x}^{1}\left(\frac{1}{x}-\frac{1}{z}\right) D_{g}(z) d z
$$

which may be computed from the measured values of $F_{L}(x)$ and $F_{T}(x)$ given in table 1 . The integration of $F_{T}(z) / z$ was performed by taking the average value of $1 / z$ across each bin from JETSET. The value of $\alpha_{s}$ was taken to be that satisfying the leading order QCD prediction for the integrals of the fragmentation functions, i.e. $\sigma_{L} / \sigma_{T}=\alpha_{s} / \pi$, and thus $\alpha_{s}=0.190$. This procedure may be expected to account crudely for unknown higher order effects ${ }^{\S}$. The gluon fragmentation function $D_{g}(z)$ is then parametrized by the form

$$
D_{g}(z)=A e^{-c \ln ^{2} z} z^{p}(1-z)^{q}
$$

This functional form may be justified by noting that it fits both $F_{T}(x)$ and $F_{L}(x)$ satisfactorily in the region $x_{E}>0.02$. The exponential term is motivated by the leading log prediction of

$\S$ This could alternatively be viewed as making a choice of the renormalization scale $\mu=x_{\mu} E_{c m}$ with $x_{\mu}=0.105$, which would yield $\alpha_{s}\left(M_{\mathrm{Z}^{0}}\right)=0.123$ when evolved to the $\mathrm{Z}^{0}$ mass scale. 
a Gaussian spectrum in $\ln 1 / z$ [22], which is observed experimentally to be a good approximation [23]. We then fit the experimental distribution of $F_{g}(x)$ to infer $D_{g}(z)$. The fit is performed over the region $0.02<x_{E}<1$, and a good fit with $\chi^{2} /$ d.o.f. $=1.07$ is obtained. The fitted parameters are $A=0.0036 \pm 0.0003, c=0.81 \pm 0.01, p=-5.89 \pm 0.04$ and $q=-0.2 \pm 0.3$, where the errors are purely statistical ". The measured distribution of $F_{g}(x)$ together with the fitted function are shown in Fig. 6. For $x_{E}>0.5$ and for the bin $0.25<x_{E}<0.3$ the measured values of $F_{g}(x)$ prove to be slightly negative, though compatible with zero within the statistical errors; we include them in the fit nonetheless. The fitted curve passes above the data for $x_{E}<0.02$; a satisfactory value of $\chi^{2} /$ d.o.f. is not obtained if these points are included in the fit.

The distribution of $D_{g}(z)$ extracted from this fit is shown in Fig. 7, and tabulated in Table 3. For convenience of presentation the fitted function has been binned in the same way as the fragmentation functions. Contributions to the systematic errors on $D_{g}(z)$ were estimated by repeating the extraction of $D_{g}(z)$ for the $F_{T}(x)$ and $F_{L}(x)$ distributions obtained in each of the systematic checks described in Sect. 3. The changes in $D_{g}(z)$ in each bin were then combined in the same way as the systematic errors on $F_{T}(x)$ and $F_{L}(x)$ to yield the overall uncertainties shown in Fig. 7, which are consequently highly correlated. In the case of some of the systematic checks, the fitted parameters describing $D_{g}(z)$ are considerably different (for example the most extreme set has $A=0.050, c=0.53, p=-4.29$ and $q=3.9)$, and consequently the systematic uncertainties become large for $z<0.05$, and also for large values of $z$. However, in the region roughly $0.05<z<0.25$ the fits give generally consistent results for $D_{g}(z)$.

For comparison we also show in Fig. 7 the predictions of the JETSET model for the fragmentation of gluon jets, generated as back-to-back gluon pairs at centre-of-mass energies 91.2 and $20 \mathrm{GeV}$, thus corresponding to gluons of 45.6 and $10 \mathrm{GeV}$. The effect of scaling violations is clearly seen in the Monte Carlo. However, the calculations of Ref. [2] to $\mathcal{O}\left(\alpha_{s}\right)$, which we are using here to extract $D_{g}(z)$, do not incorporate scaling violations, and therefore the corresponding energy scale for this measurement is not well defined. OPAL has also studied gluon jet fragmentation in Ref. $[5,6]$ using tagged three-jet events, However, a direct comparison with those data is not yet possible, since the data of Ref. $[5,6]$ were not corrected for detector response, because the main objective of those analyses was to establish the existence of quark-gluon differences in a model independent fashion.

\subsection{The Asymmetry Fragmentation Function}

In discussing Fig. 4 we noted that JETSET and HERWIG modelled the asymmetry fragmentation function $F_{A}^{c h}(x)$ reasonably well. The general structure of a positive asymmetry for $x_{E}$ less than 0.05 , with a negative asymmetry at higher $x_{E}$ is well reproduced by both models. As discussed in Ref. [24], at large values of $x_{E}$ the produced hadron is likely to contain the primary quark from the $Z^{0}$ decay, and thus preferentially to have the same sign of charge. Allowing for the forward-backward asymmetries resulting from parity violation in the weak interactions, one

IThe fitted value of $q$ is negative, though consistent with zero. This leads to an unphysical divergence in $D_{g}(z)$ as $z \rightarrow 1$. An alternative parametrization, using the function $A z^{p}(1-z)^{q}$, i.e. omitting the exponential term, fits the data slightly less well, with $\chi^{2} /$ d.o.f. $=1.44$, yielding $A=13.9 \pm 8.5, p=-0.85 \pm 0.18$ and $q=9.2 \pm 1.4$. This parametrization of $D_{g}(z)$ agrees within errors with our quoted result, and falls to zero at large $z$. 
would thus expect to see a negative value of $F_{A}$ for d-, s- and b-quark events, and a positive value for $F_{A}$ for u- and c-quark events, at large $x_{E}$. These expectations are confirmed by Monte Carlo studies. Since d-, s- and b-quarks are produced more copiously than u- and c-quarks in $\mathrm{Z}^{0}$ decays, the overall $F_{A}$ is negative, as observed. According to the Monte Carlo models, the change in sign of $F_{A}$ at $x_{E}<0.05$ is associated with $D^{*+} \rightarrow D^{0} \pi^{+}$decays in c- and b-quark events. In this decay, the small energy release forces the pion to small $x_{E}$, thus providing a mechanism whereby the charge of a pion at low $x_{E}$ is correlated with the primary quark charge in the case of $c \rightarrow D^{*+} \rightarrow D^{0} \pi^{+}$and anticorrelated in the case of $b \rightarrow c \rightarrow D^{*+} \rightarrow D^{0} \pi^{+}$, hence generating a positive asymmetry for both c- and b-quark events.

We show in Figure 8 the experimental values for the ratio $R \equiv F_{A}(x) /\left(F_{T}(x)+F_{L}(x)\right)$; we note that the magnitude of $R$ grows with $x_{E}$, indicating that information about the primary quark charge tends to be most strongly retained by the large $x_{E}$ particles. In Ref. [24] predictions are made for the ratio $R$ in the "valence dominance" approximation, i.e. under the assumption that a high $x_{E}$ hadron contains one of the primary quarks. Monte Carlo studies in Ref. [24] suggest that this approximation may be reasonable for $x_{E}>\mathbf{0 . 6}$. Under this assumption, a $\pi^{+}$at high $x_{E}$ will originate from a primary $\mathrm{u}^{-}$or $\overline{\mathrm{d}}$-quark, and the ratio $R_{\pi}$ is predicted to be -0.034 for a weak mixing angle $\sin ^{2} \theta_{W}=0.2322$. We may apply a similar argument to $K^{+}$production, which may arise from a primary u- or $\overline{\mathrm{s}}$-quark, but with the formation from a u-quark suppressed by a factor $\lambda$ representing the reduced probability of exciting an $\overline{\mathrm{s}}$-quark from the sea. In the JETSET model, a value of $\lambda \sim 0.30 \pm 0.05$ is able to account for strange meson production, in which case we would expect $R_{K}=-0.090 \mp 0.006$ (the error coming from the assumed uncertainty in $\lambda$ ). Applying a similar argument to proton production yields $R_{p}=+0.116$ [24]. OPAL measurements of charged hadron production [25] indicate that $\pi^{+}$, $K^{+}$and protons are produced in the ratios $55 \pm 3 \%: 40 \pm 3 \%: 5 \pm 1 \%$ for $x_{E}>0.44$, and thus we would predict $R=-0.049 \pm 0.004$, shown as a shaded band in Fig. 8 . The data in Fig. 8 are clearly compatible with this simple model at large $x_{E}$; integrating the data from table 1 over the region $x_{E}>0.6$ yields $R=-0.049 \pm 0.014$.

\section{Discussion and Summary}

We have measured the longitudinal, transverse and asymmetry fragmentation functions for charged particles using OPAL data on hadronic $\mathrm{Z}^{0}$ decays. The integrated longitudinal cross-

section is expected (to leading order in QCD) to be $\left(\frac{\alpha_{s}}{\pi}\right) \sigma_{0}$, where $\sigma_{0}$ is the Born cross-section, i.e. the hadronic cross-section in the absence of QCD radiation. It is therefore a small part of the total cross-section, which may be identified from its influence on the angular distribution of hadrons.

The measurement therefore relies on high statistics and good control of the systematic errors on the angular distributions of produced hadrons. Using 3.06 million events from the 19901994 OPAL data sample, we find that the statistics permit a measurement of the longitudinal fragmentation function up to $x_{E} \sim 0.5$, and of the longitudinal cross-section with a statistical precision of about $1 \%$. However, the high statistics data reveal significant systematic effects in the $\cos \theta$ distributions at the $\sim 1 \%$ level, which are not reproduced by the Monte Carlo detector simulation program. We estimate the possible influence of these problems by changing 
the fit regions or analysis cuts, and conservatively estimate the systematic uncertainty on the longitudinal cross-section to be around $9 \%$. When these systematic effects are taken into account, a useful measurement of the longitudinal fragmentation function may be made out to $x_{E} \sim 0.3$. By integrating the fragmentation functions, we determine the average charged particle multiplicity to be $\overline{n_{c h}}=21.05 \pm 0.01 \pm 0.20$, where the first error is statistical and the second systematic.

We find that the measured value of the longitudinal to total cross-section ratio,

$$
\sigma_{L} / \sigma_{t o t}=0.057 \pm 0.005
$$

agrees well with the expectations of the JETSET and HERWIG parton shower models. However, it lies considerably above the $\mathcal{O}\left(\alpha_{s}\right)$ QCD prediction of $0.038 \pm 0.002$. It appears from the Monte Carlo studies that slightly less than half of this difference can be attributed to hadronization effects and the remainder to $\mathcal{O}\left(\alpha_{s}^{2}\right)$ and higher order perturbative effects.

Using the leading order QCD calculations, it is possible to extract the gluon fragmentation function $D_{g}(z)$ from the measurements of $F_{L}(x)$ and $F_{T}(x)$. Given the present experimental errors, it proves possible to make a reasonable measurement in the region approximately $0.05<$ $z<0.25$. OPAL has also investigated gluon fragmentation using three-jet events in which the less energetic quark is tagged via a heavy flavour decay $[5,6]$. However, in those studies the assignment of hadrons to jets has potentially some ambiguities, since it depends on the use of a jet finder. The present analysis therefore offers a useful complementary approach which does not depend on jet finding.

The measurements of the asymmetry fragmentation function are in agreement with QCD models. The asymmetry at large $x_{E}$ can be understood in terms of the electroweak asymmetries in quark production from $\mathrm{Z}^{0}$ decays, and the expectation that hadrons at high $x_{E}$ will tend to contain a primary quark.

\section{Acknowledgements}

We thank B.R. Webber for enlightening discussions concerning parts of this analysis.

It is a pleasure to thank the SL Division for the efficient operation of the LEP accelerator, and their continuing close cooperation with our experimental group. In addition to the support staff at our own institutions we are pleased to acknowledge the

Department of Energy, USA,

National Science Foundation, USA,

Particle Physics and Astronomy Research Council, UK,

Natural Sciences and Engineering Research Council, Canada, Fussefeld Foundation,

Israel Ministry of Science,

Israel Science Foundation, administered by the Israel Academy of Science and Humanities, Minerva Gesellschaft,

Japanese Ministry of Education, Science and Culture (the Monbusho) and a grant under the Monbusho International Science Research Program,

German Israeli Bi-national Science Foundation (GIF), 
Direction des Sciences de la Matière du Commissariat à 1'Energie Atomique, France, Bundesministerium für Forschung und Technologie, Germany, National Research Council of Canada,

A.P. Sloan Foundation and Junta Nacional de Investigação Científica e Tecnológica, Portugal.

\section{References}

[1] B.R. Webber, J. Phys. G17 (1991) 1579.

[2] P. Nason and B.R. Webber, Nucl. Phys. B421 (1994) 473.

[3] Delphi Collaboration, P. Abreu et al., Phys. Lett. B311 (1993) 408.

[4] G. Altarelli, Phys. Rep. 81 (1982) 1;

B. Mele and P. Nason, Nucl. Phys. B361 (1991) 626.

[5] Opal Collaboration, P.D. Acton et al., Z. Phys. C58 (1993) 387.

[6] Opal Collaboration, G. Alexander et al., Phys. Lett. B265 (1991) 462.

[7] Opal Collaboration, K. Ahmet et al., Nucl. Instr. and Meth. A305 (1991) 275.

[8] P.P. Allport et al., Nucl. Instr. and Meth. A324 (1993) 34;

P.P. Allport et al., Nucl. Instr. and Meth. A346 (1994) 476.

[9] D.G. Charlton, F. Meijers, T.J. Smith and P.S. Wells, Nucl. Instr. and Meth. A325 (1993) 129.

[10] Opal Collaboration, G. Alexander et al., Z. Phys. C52 (1991) 175.

[11] S. Brandt et al., Phys. Lett. 12 (1964) 57;

E. Farhi, Phys. Rev. Lett. 39 (1977) 1587.

[12] T. Sjöstrand, Comp. Phys. Commun. 39 (1986) 347;

T. Sjöstrand, Comp. Phys. Commun. 43 (1987) 367;

M. Bengtsson and T. Sjöstrand, Nucl. Phys. B289 (1987) 810.

[13] J. Allison et al., Nucl. Instr. and Meth. A317 (1992) 47.

[14] G. Marchesini and B.R. Webber, Nucl. Phys. B310 (1988) 461;

G. Marchesini et al., Comp. Phys. Commun. 67 (1992) 465.

[15] Opal Collaboration, P.D. Acton et al., Z. Phys. C53 (1992) 539.

[16] Opal Collaboration, M.Z. Akrawy et al., Z. Phys. C47 (1990) 505.

[17] T. Hebbeker, M. Martinez, G. Passarino and G. Quast, Phys. Lett. B331 (1994) 165.

[18] Particle Data Group, L. Montanet et al., Phys. Rev. D50 (1994) 1173.

[19] Opal Collaboration, P.D. Acton et al., Z. Phys. C59 (1993) 1. 
[20] B.R. Webber, Phys. Lett. B339 (1994) 148.

[21] Yu.L. Dokshitzer and B.R. Webber, Preprint Cavendish-HEP-95/2; LU TP 95-8.

[22] Yu.L. Dokshitzer, V.A.Khoze, A.H.Mueller and S.I.Troyan, Basics of Perturbative QCD (Editions Frontières, Paris) 1991.

[23] Opal Collaboration, M.Z. Akrawy et al., Phys. Lett. B247 (1990) 617.

[24] P. Nason and B.R. Webber, Phys. Lett B332 (1994) 405.

[25] Opal Collaboration, R. Akers et al., Z. Phys. C63 (1994) 181. 


\begin{tabular}{|c|c|c|}
\hline 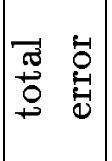 & 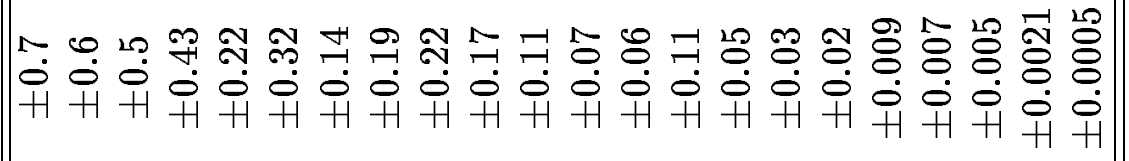 & 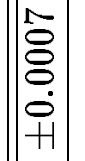 \\
\hline 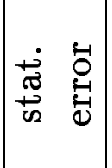 & 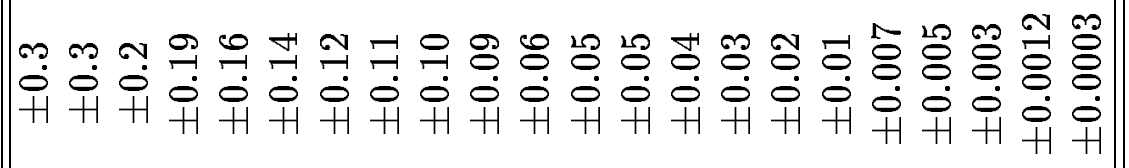 & 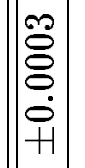 \\
\hline$\underbrace{8}_{[-8}$ & 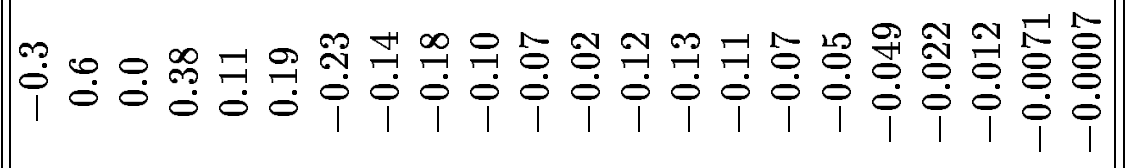 & 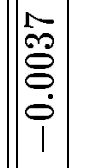 \\
\hline 8 & 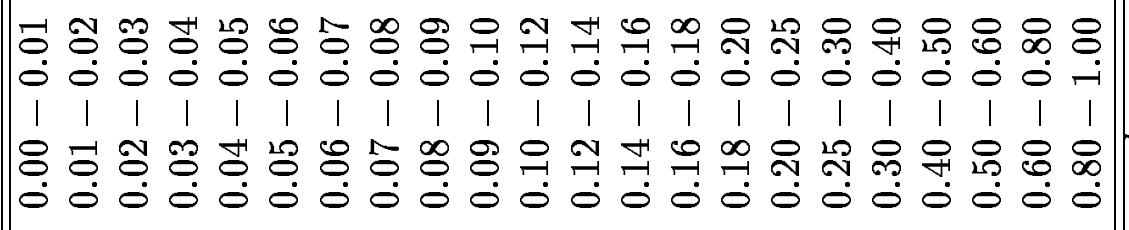 & $\mid \begin{array}{c}\overrightarrow{0} \\
\vec{b} \\
\vec{s} \\
b \\
b\end{array}$ \\
\hline 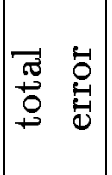 & 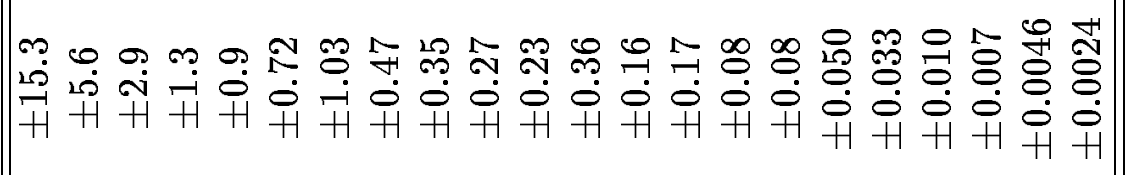 & 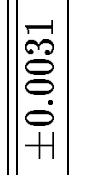 \\
\hline 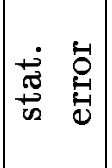 & 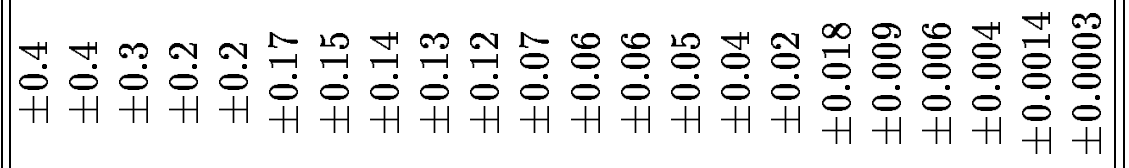 & 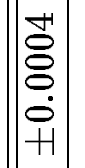 \\
\hline$\overbrace{5}^{8}$ & 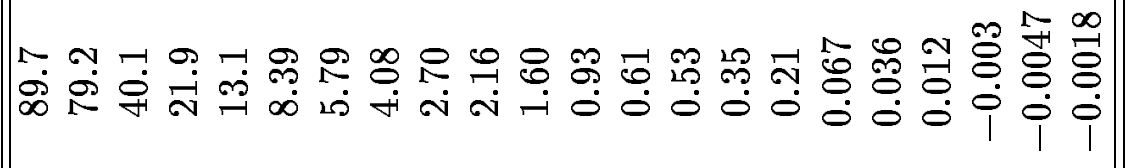 & 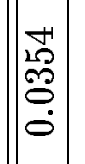 \\
\hline \&े & 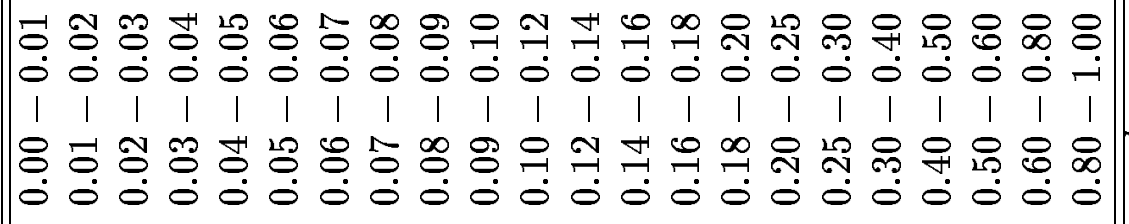 & 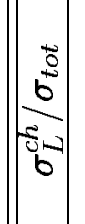 \\
\hline 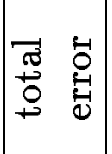 & 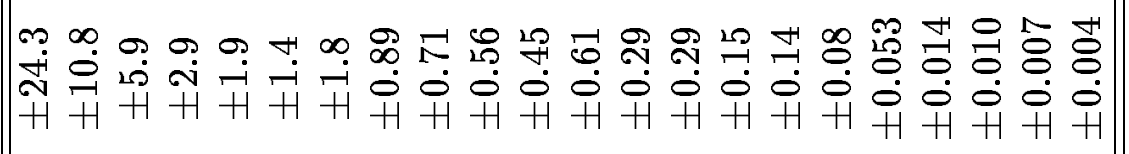 & $\mid \begin{array}{l}\mathscr{Q} \\
\mathscr{O} \\
\dot{0} \\
\dot{H} \\
+1\end{array}$ \\
\hline 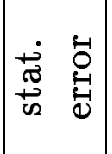 & 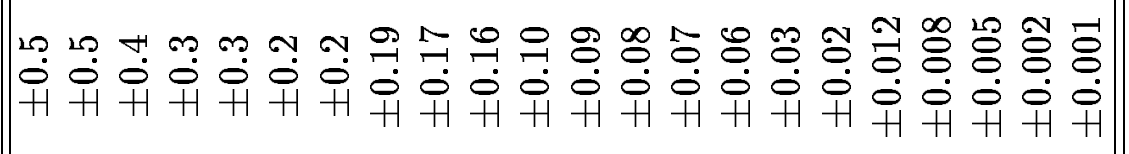 & $\mid \begin{array}{l}\mathscr{\rho} \\
\stackrel{8}{\odot} \\
\dot{0} \\
+1\end{array}$ \\
\hline 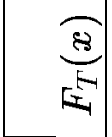 & 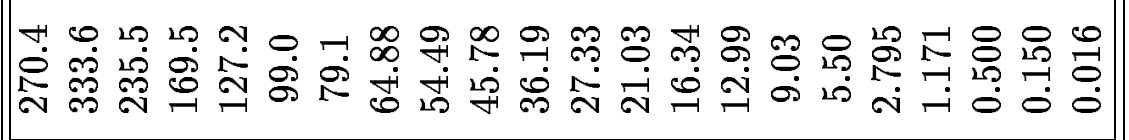 & $\mid \begin{array}{c}\infty \\
\infty \\
2 \\
1 \\
0\end{array}$ \\
\hline$\$$ & 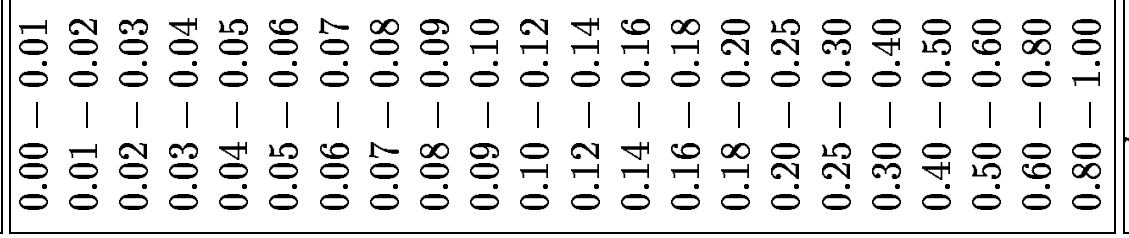 & 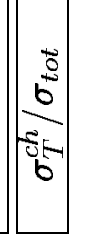 \\
\hline
\end{tabular}




\begin{tabular}{|c|c|c|c|}
\hline Central values: & $\frac{\sigma_{\boldsymbol{T}}^{c h}}{\sigma_{t o t}}=\mathbf{0 . 5 8 8 0}$ & $\frac{\sigma_{L}^{c h}}{\sigma_{t o t}}=0.0354$ & $\frac{\sigma_{A}^{c h}}{\sigma_{t o t}^{c}}=-\mathbf{0 . 0 0 3 7}$ \\
\hline Error contribution & $\Delta \sigma_{T}^{c h} / \sigma_{t o t}$ & $\Delta \sigma_{L}^{c h} / \sigma_{t o t}$ & $\Delta \sigma_{A}^{c h} / \sigma_{t o t}$ \\
\hline Statistical & \pm 0.0005 & \pm 0.0004 & \pm 0.0003 \\
\hline$|\cos \theta|<0.60$ or $0.8<|\cos \theta|<0.88$ & -0.0026 & +0.0015 & +0.0002 \\
\hline$|\cos \theta|<0.64$ & +0.0017 & -0.0011 & +0.0006 \\
\hline$|\cos \theta|<0.60$ & +0.0010 & -0.0006 & +0.0006 \\
\hline$\left|d_{0}\right|<1 \mathrm{~cm}$ & +0.0008 & +0.0010 & 0.0000 \\
\hline$\left|d_{0}\right|<5 \mathrm{~cm}$ & -0.0008 & -0.0004 & 0.0000 \\
\hline$\left|z_{0}\right|<10 \mathrm{~cm}$ & -0.0040 & +0.0009 & 0.0000 \\
\hline$p_{T}>0.15 \mathrm{GeV} / \mathrm{c}$ & -0.0002 & +0.0001 & 0.0000 \\
\hline$n_{C J} \geq 40$ & +0.0015 & -0.0008 & 0.0000 \\
\hline$\left|\cos \theta_{\text {Thrust }}\right|<0.9$ & -0.0028 & +0.0021 & +0.0001 \\
\hline No presampler info. & -0.0009 & +0.0007 & -0.0002 \\
\hline Use silicon $\mathrm{z}$ (where available) & -0.0003 & +0.0002 & +0.0001 \\
\hline Total (statistical+systematic) & \pm 0.0060 & \pm 0.0031 & $\pm \mathbf{0 . 0 0 0 7}$ \\
\hline
\end{tabular}

Table 2: Contributions to the errors on the transverse, longitudinal and asymmetry crosssections; the sign gives the direction of change when the analysis was altered. 


\begin{tabular}{|c||c|}
\hline$z$ & $D_{g}(z)$ \\
\hline \hline $0.02-0.03$ & $152 \pm 168$ \\
$0.03-0.04$ & $146 \pm 82$ \\
$0.04-0.05$ & $125 \pm 40$ \\
$0.05-0.06$ & $102 \pm 22$ \\
$0.06-0.07$ & $82 \pm 14$ \\
$0.07-0.08$ & $66.0 \pm 9.4$ \\
$0.08-0.09$ & $52.9 \pm 6.6$ \\
$0.09-0.10$ & $42.6 \pm 4.9$ \\
$0.10-0.12$ & $31.2 \pm 3.3$ \\
$0.12-0.14$ & $20.9 \pm 1.7$ \\
$0.14-0.16$ & $14.3 \pm 1.0$ \\
$0.16-0.18$ & $9.95 \pm 0.84$ \\
$0.18-0.20$ & $7.08 \pm 0.80$ \\
$0.20-0.25$ & $4.16 \pm 0.73$ \\
$0.25-0.30$ & $2.02 \pm 0.59$ \\
$0.30-0.40$ & $0.82 \pm 0.38$ \\
$0.40-0.50$ & $0.28 \pm 0.20$ \\
$0.50-0.60$ & $0.11 \pm 0.11$ \\
$0.60-0.80$ & $0.04 \pm 0.05$ \\
$0.80-1.00$ & $0.01 \pm \mathbf{0 . 0 3}$ \\
\hline
\end{tabular}

Table 3: Values of the gluon fragmentation function, $D_{g}(z)$, inferred from the longitudinal and transverse fragmentation functions. The errors include statistical and systematic contributions, and are highly correlated. The data are presented in Fig. 7. 

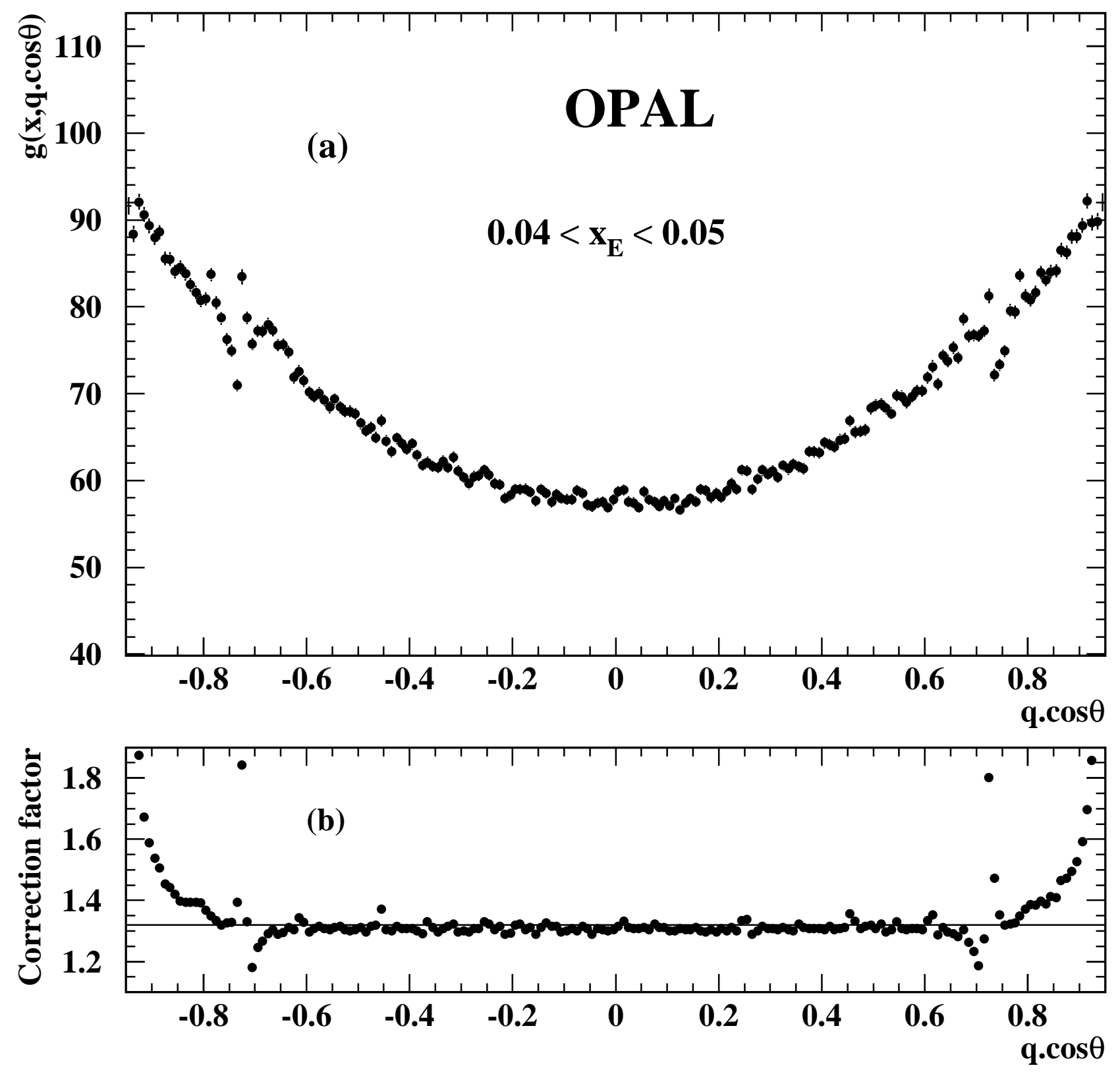

Figure 1: (a) Corrected $q \cdot \cos \theta$ distribution, $g(x, q \cdot \cos \theta)$ for tracks having $0.04<x_{E}<0.05$. (b) The corresponding correction factors. The line shows the level expected on the basis of the azimuthal angle cuts applied. 

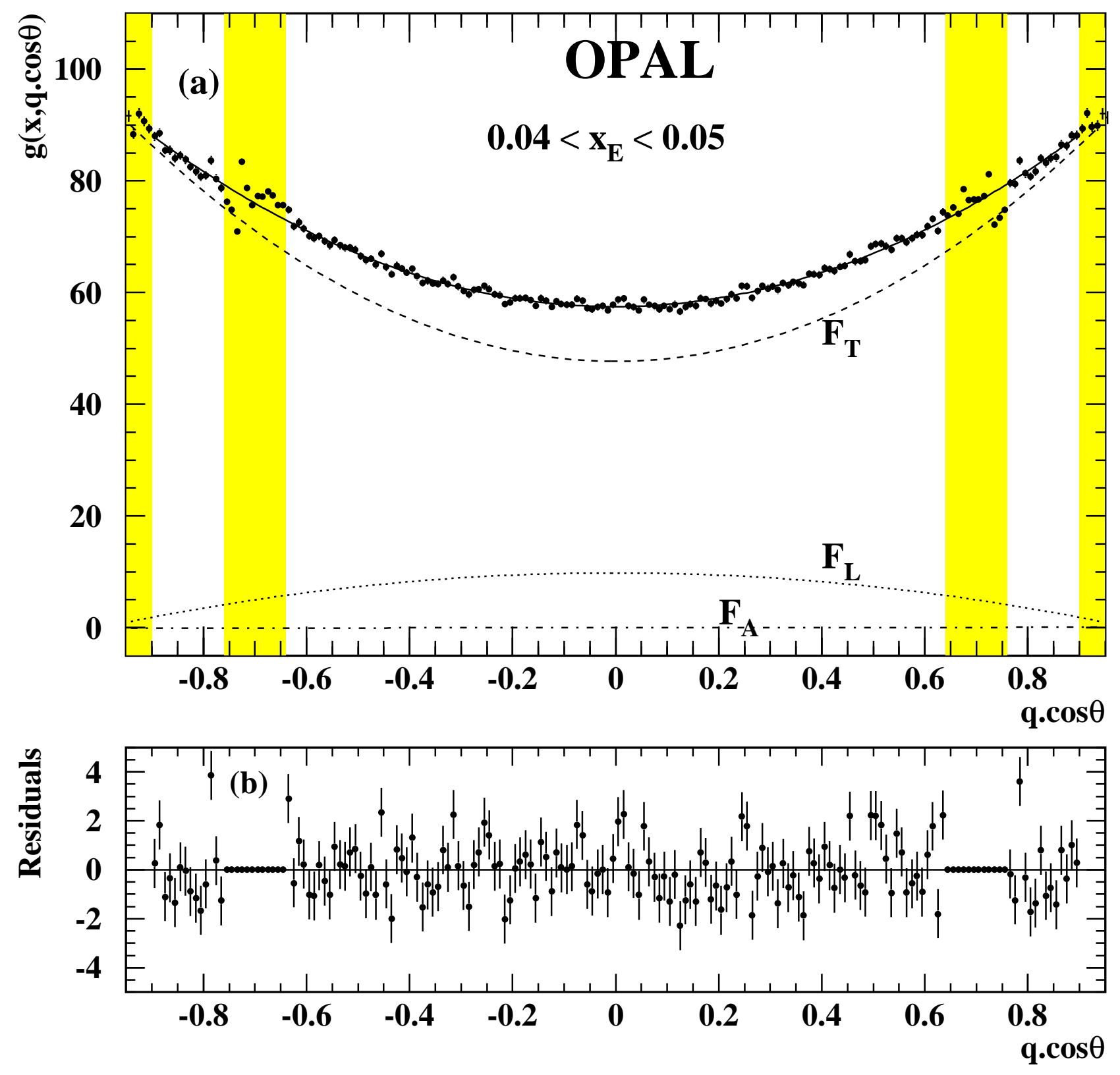

Figure 2: (a) Fit to the corrected $q \cdot \cos \theta$ distribution, $g(x, q \cdot \cos \theta)$ for tracks having $0.04<$ $x_{E}<0.05$. The shaded regions are excluded from the fit. The fitted contributions shown are: transverse (dashed), longitudinal (dotted), asymmetry (dot-dashed) and total (solid). (b) Residuals of the fit. 

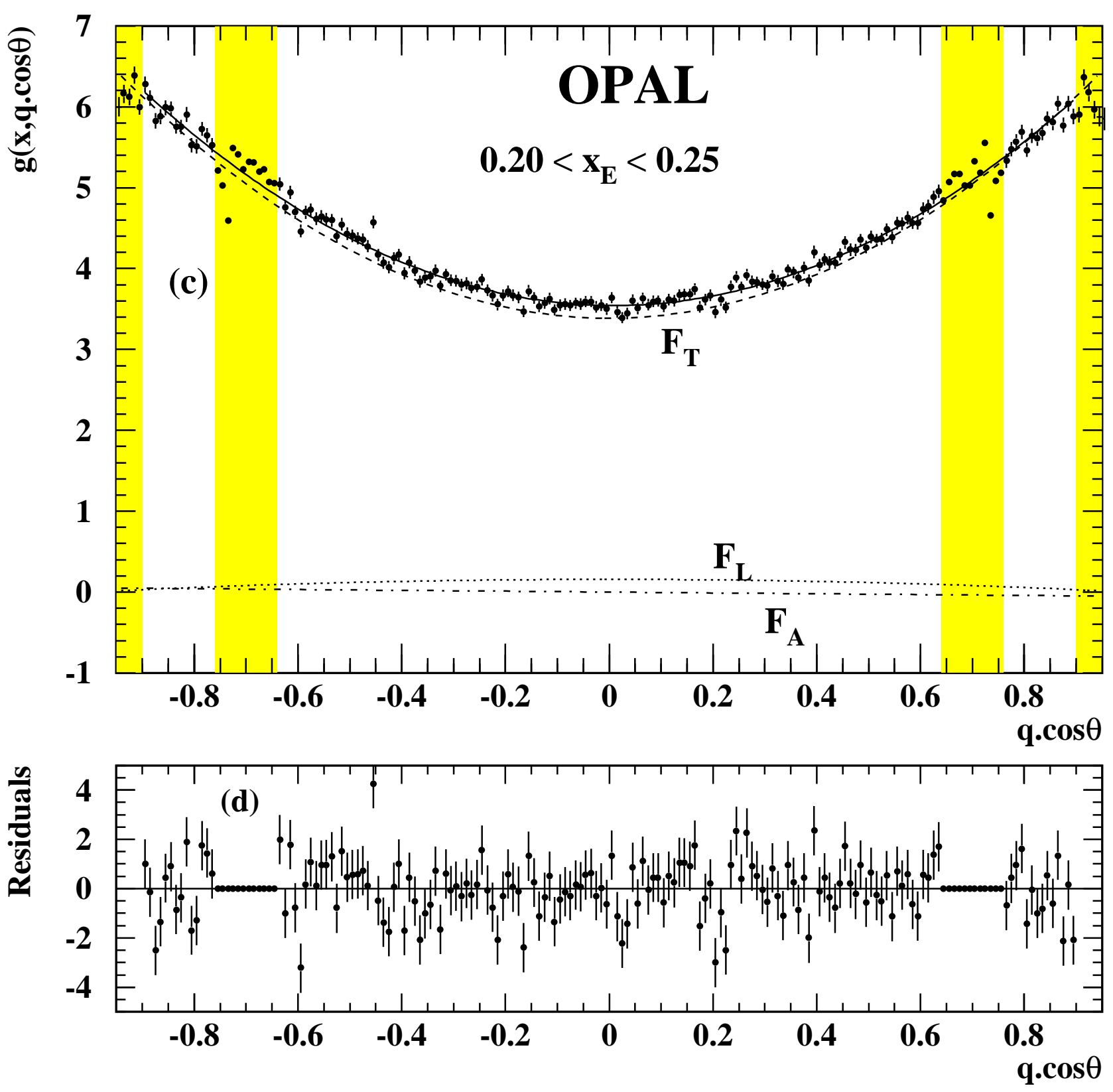

Figure 2: (c) as (a), for tracks having $0.20<x_{E}<0.25$. (d) Residuals of the fit. 


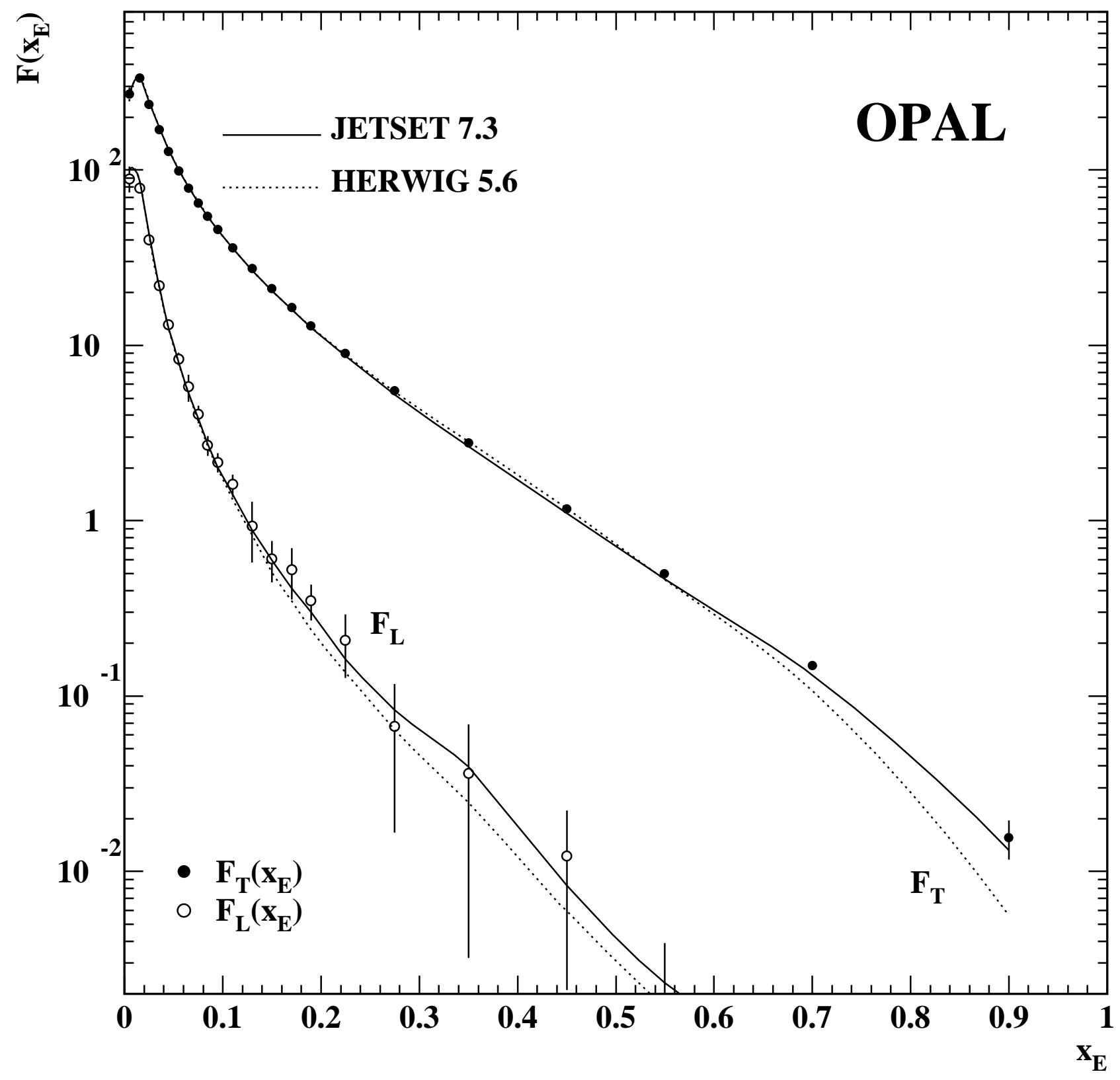

Figure 3: Measurements of $F_{T}(x)$ and $F_{L}(x)$ for charged particles; statistical and systematic errors are combined. The predictions of the QCD Monte Carlo programs JETSET and HERWIG are also shown. The data points are plotted at the bin centres, and the model predictions are averaged over the same bins and drawn as curves passing through the bin centres. 


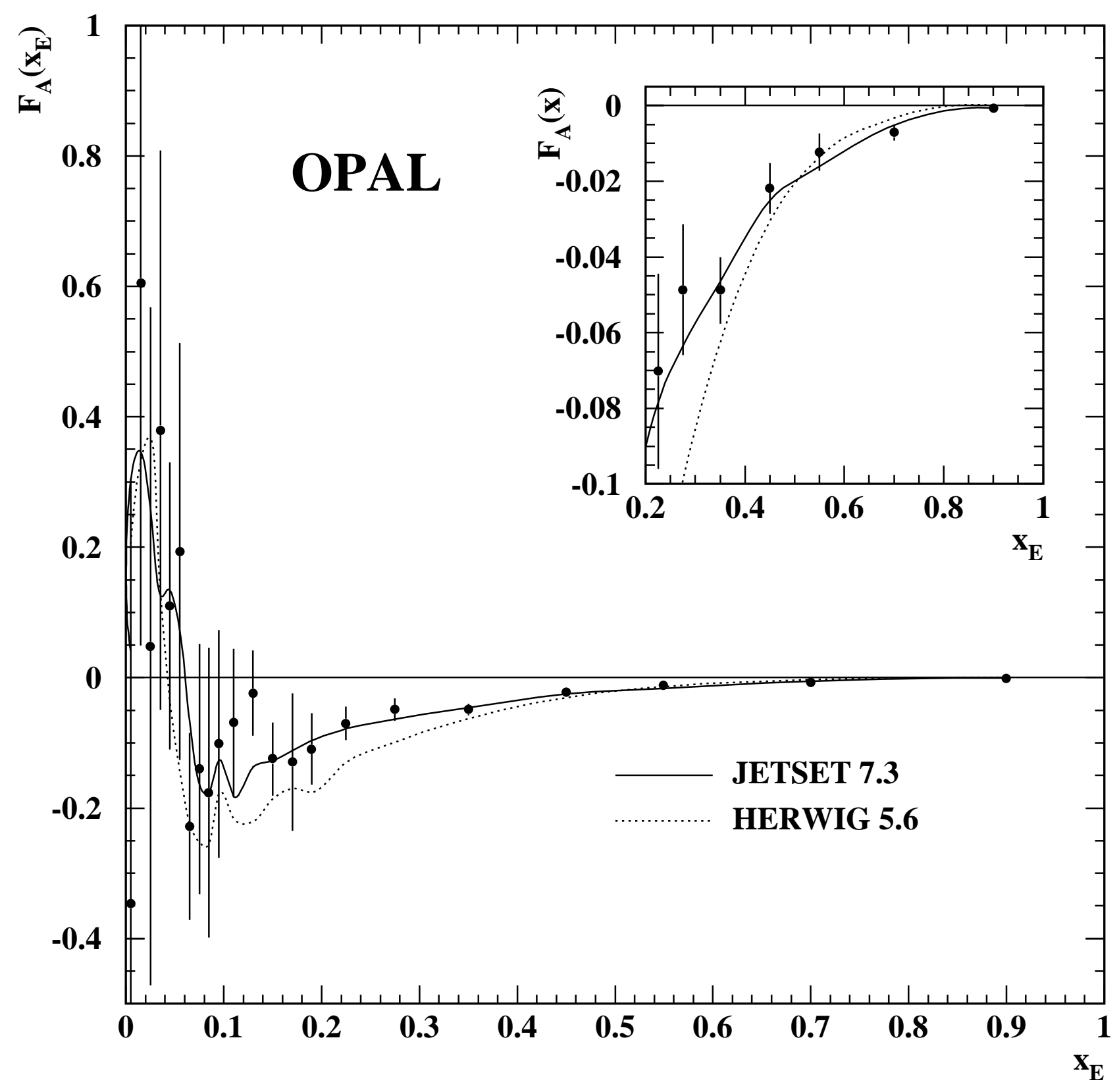

Figure 4: The Asymmetric component of the fragmentation function, $F_{A}(x)$ derived from the OPAL data. The inset shows the high $x_{E}$ region on an expanded scale. The predictions of the QCD Monte Carlo programs JeTSET and HERWIG are also shown. 


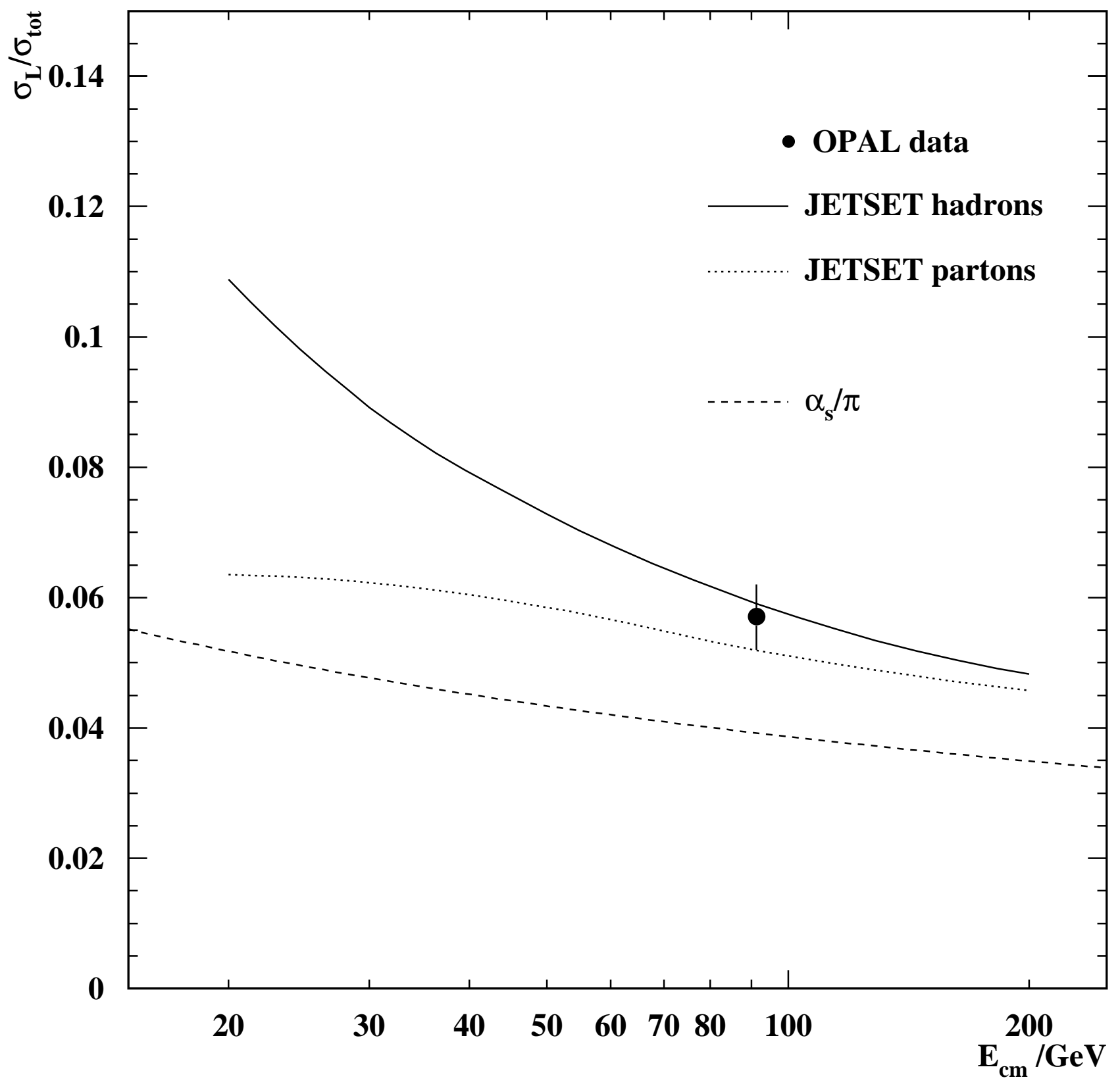

Figure 5: Dependence of $\sigma_{L} / \sigma_{t o t}$ on centre-of-mass energy $E_{c m}$ as predicted by the JETSET model. The predictions are shown for the parton shower model both for the hadrons and for the partons at the end of the parton shower. The leading order prediction $\alpha_{s} / \pi$ is also shown, taking $\alpha_{s}=0.123$. 


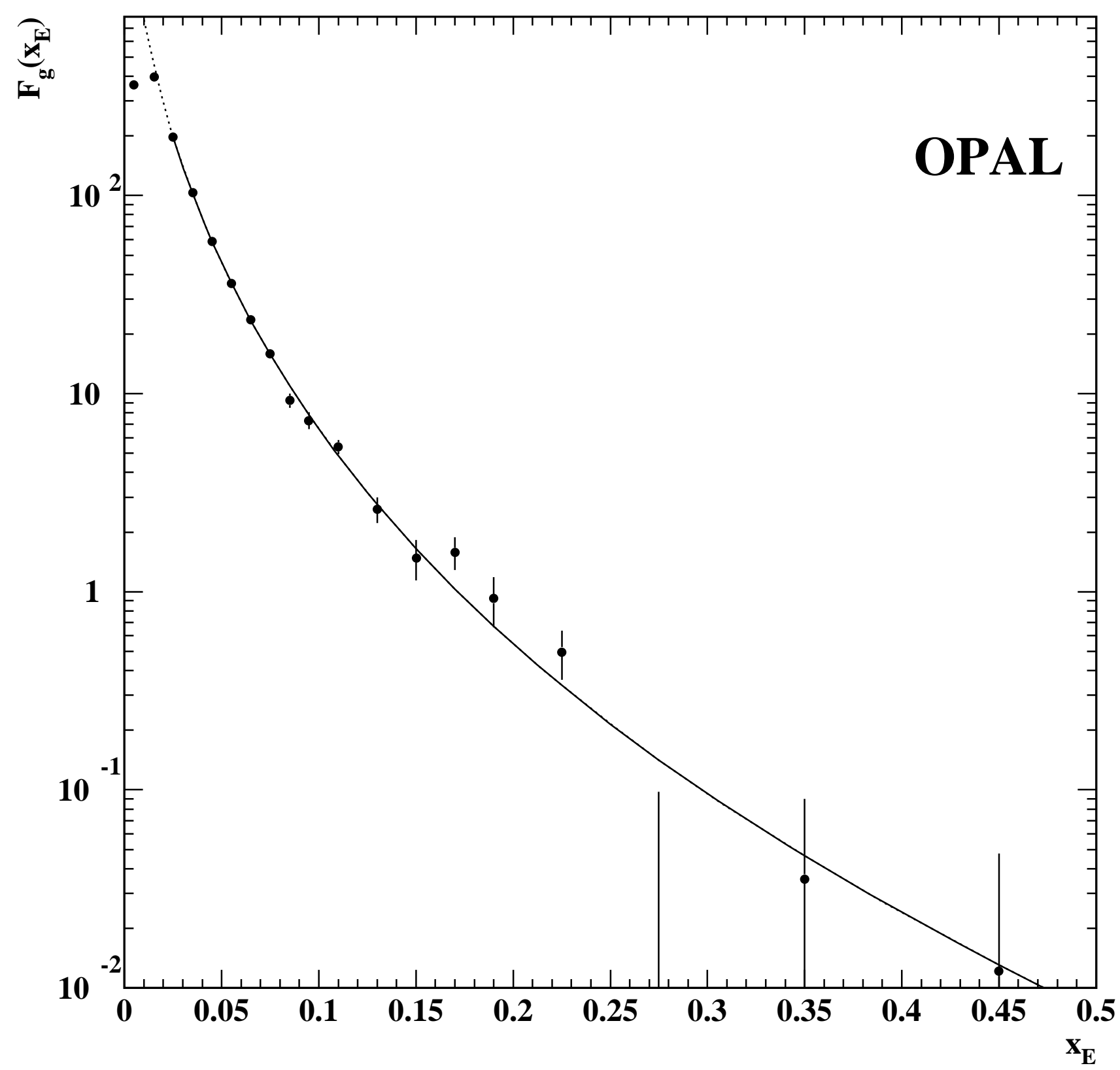

Figure 6: Distribution of $F_{g}(x)=\frac{\pi}{2 \alpha_{s} C_{F}} F_{L}(x)-\frac{1}{4} \int_{x}^{1} \frac{F_{T}(z)}{z} d z$. The curve shows a fit to the form $\int_{x}^{1}\left(\frac{1}{x}-\frac{1}{z}\right) D_{g}(z) d z$, from which $D_{g}(z)$ may be inferred. The solid part of the curve indicates the fitted region; the extrapolation to low $x_{E}$ is shown as a dotted line. 


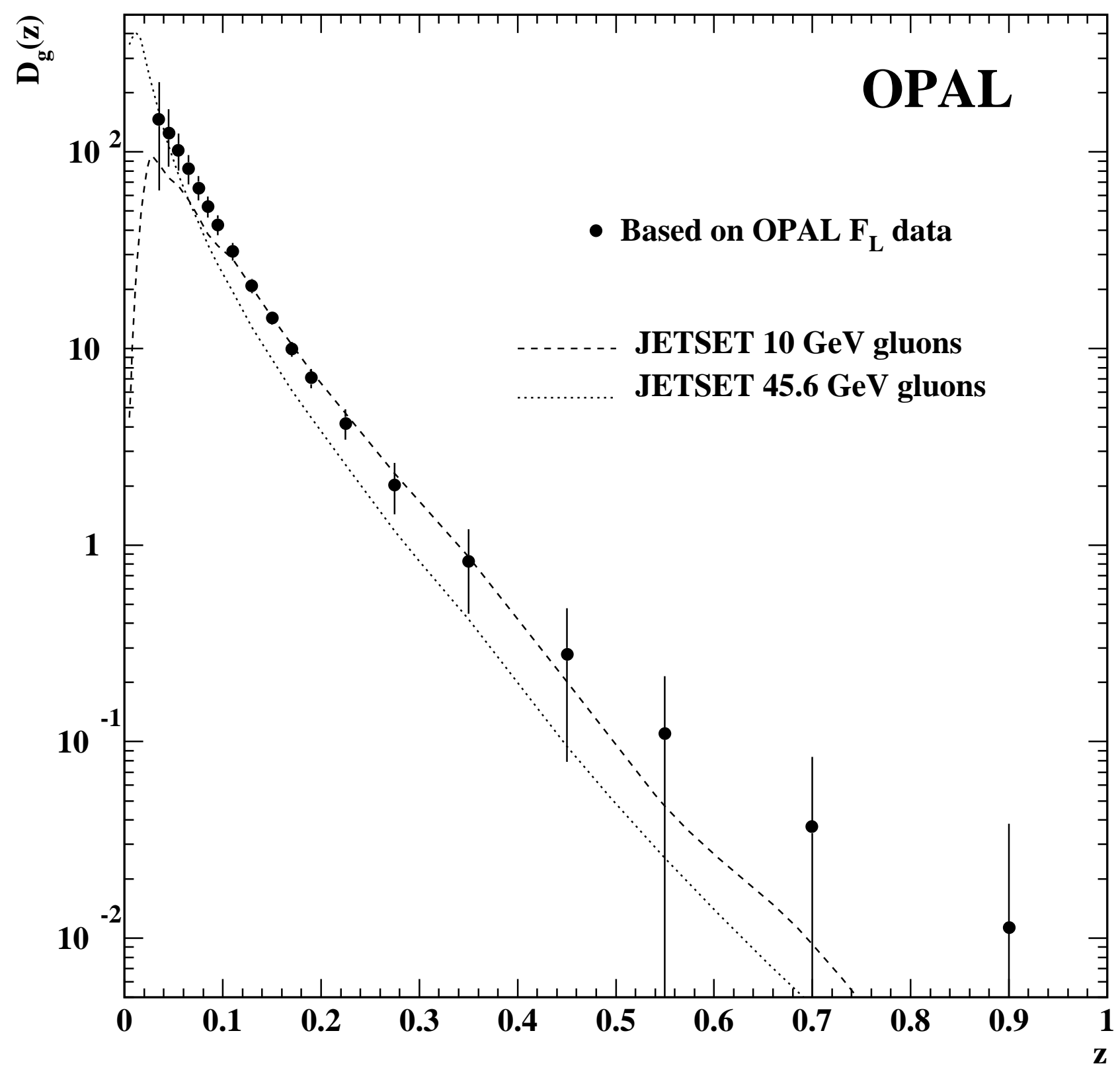

Figure 7: Gluon fragmentation function $D_{g}(z)$ for charged particles extracted from the longitudinal and transverse fragmentation functions. The errors include systematic contributions, and are highly correlated. Numerical values are given in Table 3 . The predictions of the JETSET model for the fragmentation of gluon jets (in a gluon-gluon system) at two energies are shown. 


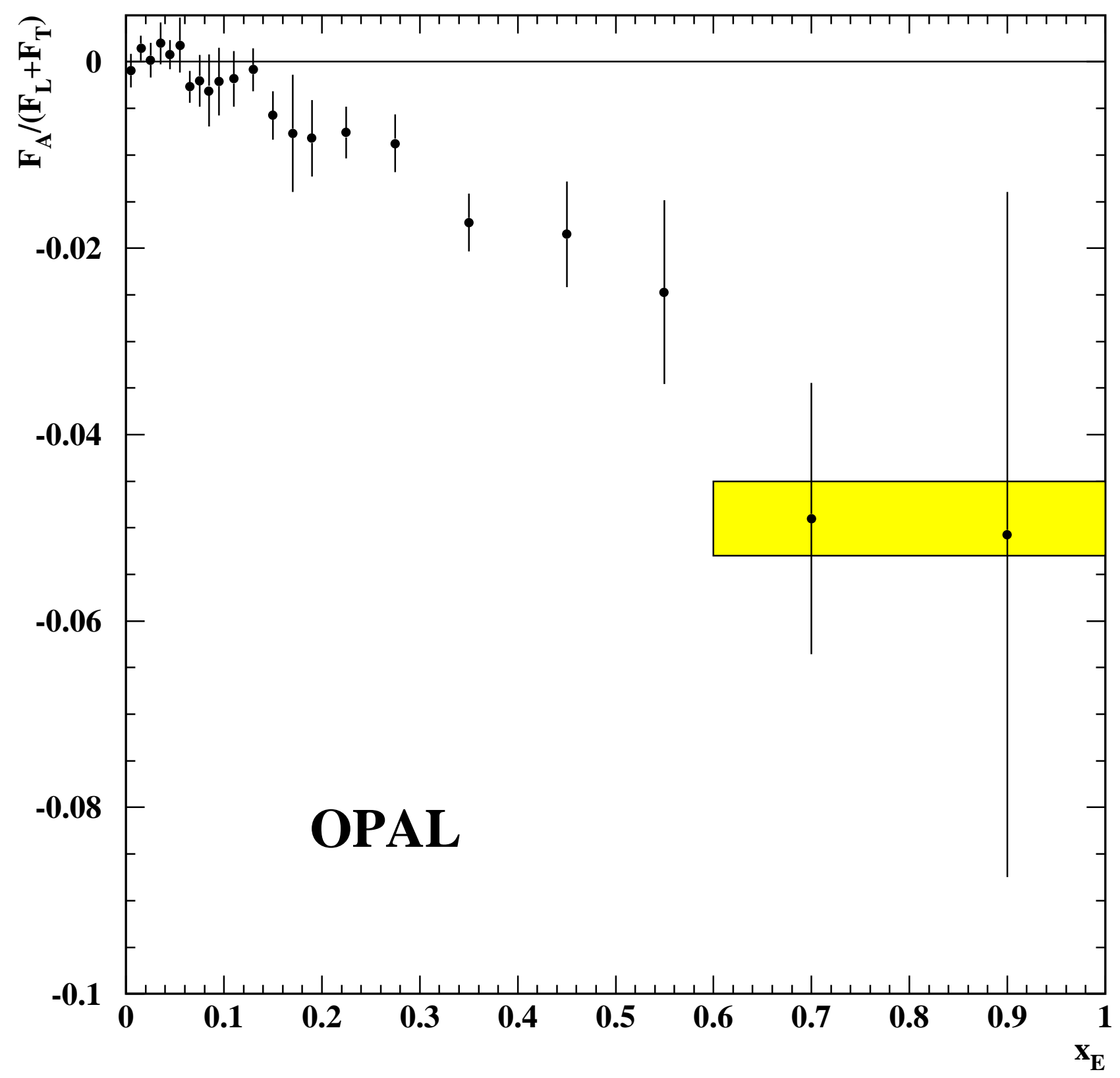

Figure 8: The asymmetry fragmentation function as a fraction of the total fragmentation function, $R \equiv F_{A}(x) /\left(F_{T}(x)+F_{L}(x)\right)$. The shaded band shows the simple theoretical prediction discussed in the text. 